\title{
Pionic deuterium
}

Th. Strauch ${ }^{1}$, F. D. Amaro ${ }^{2}$, D. F. Anagnostopoulos ${ }^{3}$, P. Bühler ${ }^{4}$, D. S. Covita ${ }^{2,7 a}$, H. Gorke ${ }^{5}$, D. Gotta ${ }^{1 b}$, A. Gruber ${ }^{4}$, A. Hirtl ${ }^{4 c}$, P. Indelicato ${ }^{6}$, E.-O. Le Bigot ${ }^{6}$, M. Nekipelov ${ }^{1}$, J. M. F. dos Santos ${ }^{2}$, Ph. Schmid ${ }^{4}$, S. Schlesser ${ }^{6}$, L. M.

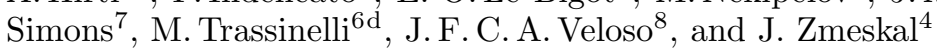

1 Institut für Kernphysik, Forschungszentrum Jülich, D-52425 Jülich, Germany

2 Dept. of Physics, Coimbra University, P-3000 Coimbra, Portugal

3 Dept. of Materials Science and Engineering, University of Ioannina, GR-45110 Ioannina, Greece

4 Stefan Meyer Institut for Subatomic Physics, Austrian Academy of Sciences, A-1090 Vienna, Austria

5 Zentralinstitut für Elektronik, Forschungszentrum Jülich GmbH, D-52425 Jülich, Germany

6 Laboratoire Kastler Brossel, UPMC-Paris 6, ENS, CNRS; Case 74, 4 place Jussieu, F-75005 Paris, France

7 Laboratory for Particle Physics, Paul Scherrer Institut, CH-5232 Villigen, Switzerland

8 I3N, Dept. of Physics, Aveiro University, P-3810 Aveiro, Portugal

Received: date / Revised version: date

\begin{abstract}
The strong-interaction shift $\epsilon_{1 s}^{\pi \mathrm{D}}$ and broadening $\Gamma_{1 s}^{\pi \mathrm{D}}$ in pionic deuterium have been determined in a high statistics study of the $\pi \mathrm{D}(3 p-1 s)$ X-ray transition using a high-resolution crystal spectrometer. The pionic deuterium shift will provide constraints for the pion-nucleon isospin scattering lengths extracted from measurements of shift and broadening in pionic hydrogen. The hadronic broadening is related to pion absorption and production at threshold. The results are $\epsilon_{1 s}^{\pi \mathrm{D}}=(-2356 \pm 31) \mathrm{meV}$ (repulsive) and $\Gamma_{1 s}^{\pi \mathrm{D}}=\left(1171_{-49}^{+23}\right) \mathrm{meV}$ yielding for the complex $\pi \mathrm{D}$ scattering length $a_{\pi \mathrm{D}}=\left[-(24.99 \pm 0.33)+i\left(6.22_{-0.26}^{+0.12}\right)\right] \times 10^{-3} m_{\pi}^{-1}$. From the imaginary part, the threshold parameter for pion production is obtained to be $\alpha=\left(251_{-11}^{+5}\right) \mu \mathrm{b}$. This allows, in addition, and by using results from pion absorption in ${ }^{3} \mathrm{He}$ at threshold, the determination of the effective couplings $g_{0}$ and $g_{1}$ for s-wave pion absorption on isoscalar and isovector $N N$ pairs.
\end{abstract}

PACS. $36.10 . \mathrm{Gv}$, 25.80.Ls, 07.85.Nc Mesonic atoms, Pion inclusive scattering and absorption, X-ray spectrometers

\section{Introduction}

Hadronic atoms reveal the influence of the strong force by a shift $\epsilon$ and broadening $\Gamma$ of the low-lying atomic levels with respect to the pure electromagnetic interaction. As atomic binding energies are negligibly small compared to the hadronic scale, a measurement of shift and width is equivalent to a scattering experiment at zero energy (threshold). Hence, such atomic data contain information on hadron-nucleus scattering lengths [1,2].

In pionic hydrogen, the atomic ground state level shift $\epsilon_{1 s}^{\pi \mathrm{H}}$ and broadening $\Gamma_{1 s}^{\pi \mathrm{H}}$ are connected to the two isospinseparated pion-nucleon $(\pi N)$ scattering lengths. In the case of nuclei $A(N, Z)$ with $A \geq 2, \epsilon_{1 s}^{\pi \mathrm{A}}$ and $\Gamma_{1 s}^{\pi \mathrm{A}}$ are measures of the complex pion-nucleus scattering length

\footnotetext{
a present address: I3N, Dept. of Physics, Aveiro University,

P-3810 Aveiro, Portugal

b corresponding author: d.gotta@fz-juelich.de

c present address: Universitätsklinik für Nuklearmedizin,

Medizinische Universität Wien, 1090 Vienna, Austria

d present address: Inst. des NanoSciences de Paris, CNRS

UMR7588 and UMPC-Paris 6, F-75015 Paris, France
}

$a_{\pi \mathrm{A}}$. The real part of $a_{\pi \mathrm{A}}$ is attributed in leading order to scattering, whereas the imaginary part is due to pion absorption inducing itself a contribution to the shift 3, 4.

It has been shown, that strong-interaction effects in low $Z$ pionic atoms can be related unambiguously to pionnucleon $(\pi N)$ scattering lengths as defined in modern field theoretical approaches of QCD [5. Such threshold quantities are of great importance because they belong to the first scattering parameters accessible by lattice calculations [6]. For the $\pi N$ case, however, results are not yet available [7,8].

Pion-nucleus dynamics is understood to be built up from elementary $\pi N \rightarrow \pi N$ and $\pi N N \leftrightarrow N N$ processes taking into account nuclear structure, multiple scattering, and absorptive phenomena. Vice versa, precise pionnucleus data could set constraints on elementary amplitudes. Generally, systematic uncertainties involved in the extraction of $a_{\pi \mathrm{A}}$ from atom data, if available, are smaller than in the extrapolation of cross-section data to threshold. In this case, normalisation problems cease to exist and corrections owing to Coulomb bound states are better under control than for scattering states (see sect.3). 
Assuming isospin conservation and charge symmetry, $\pi N \rightarrow \pi N$ scattering at threshold is described completely by two real numbers, e.g., the isoscalar and isovector $\pi N$ scattering lengths $a^{+}$and $a^{-}$. They are defined in terms of two $\pi N \rightarrow \pi N$ reactions or isospin $I=1 / 2$ and $I=3 / 2$ by

$$
\begin{aligned}
& a^{+}=\frac{1}{2}\left(a_{\pi^{-} p \rightarrow \pi^{-} p}+a_{\pi^{+} p \rightarrow \pi^{+} p}\right)=\frac{1}{3}\left(a_{1 / 2}+2 a_{3 / 2}\right), \\
& a^{-}=\frac{1}{2}\left(a_{\pi^{-} p \rightarrow \pi^{-} p}-a_{\pi^{+} p \rightarrow \pi^{+} p}\right)=\frac{1}{3}\left(a_{1 / 2}-a_{3 / 2}\right) .
\end{aligned}
$$

Furthermore, among others the relations $a_{\pi^{-} n \rightarrow \pi^{-} n}=$ $a_{\pi^{+} p \rightarrow \pi^{+} p}$ and $a_{\pi^{-} p \rightarrow \pi^{\circ} n}=-\sqrt{2} a^{-}$hold.

The scattering lengths $a^{+}$and $a^{-}$play a key role in the modern low-energy approach of QCD based on effective field theories (EFT). A perturbative method - chiral perturbation theory $(\chi \mathrm{PT})$ - has been developed, which is intimately related to the smallness of the pion's mass $m_{\pi}$ due to its underlying nature as a Goldstone boson 9 10, 11, 12. The interaction is calculated order by order as an expansion in the - compared to the hadronic scale of $1 \mathrm{GeV}$-"small" quantities momentum, pion mass, fine structure constant thus including the (isospin-breaking) electromagnetic interaction, and the light quark mass difference $m_{d}-m_{u}$ (strong isospin breaking) [13, 14, 15, 16, 17, 18, 19. In such a theory short-range contributions are summarized in so called low-energy constants (LECs) which must be determined from $\pi N$ experiments (for details see [5]).

From the scattering lengths $a^{+}$and $a^{-}$, important quantities of the $\pi N$ interaction are derived [20. The $\pi N$ $\sigma$ term $[12,21,22$ is a measure of explicit chiral symmetry breaking, and the $\pi N$ coupling constant, obtained from the Goldberger-Miyazawa-Oehme sum rule 23,24 25 , is related to the strength of threshold photo production of charged pions [26, 27, 28, and the induced pseudoscalar coupling $g_{p}$ as determined from muon capture in hydrogen [29, 30, 31].

Access to $a^{+}$and $a^{-}$is available from the $1 s$ level shift and broadening in pionic hydrogen $(\pi \mathrm{H})$ :

$$
\begin{array}{ll}
\epsilon_{1 s}^{\pi \mathrm{H}} \propto a_{\pi^{-} p \rightarrow \pi^{-}} & =a^{+}+a^{-}+\ldots \\
\Gamma_{1 s}^{\pi \mathrm{H}} \propto\left(1+\frac{1}{P}\right)\left(a_{\pi^{-} p \rightarrow \pi^{0} n}\right)^{2} & \propto\left(a^{-}\right)^{2} \quad+\ldots
\end{array}
$$

Ellipses stand for corrections owing to the fact that the pionic atom constitutes a Coulomb state as well as for electromagnetic and strong isospin and non-isospin breaking corrections, which are essential to extract $a^{+}$and $a^{-}$in a well defined way from the data. These corrections may be calculated, e.g., within the framework of $\chi \mathrm{PT}$ and are of the order of a few per cent [5, 32, 33, 34, 35.

In the $\pi \mathrm{H}$ case, the level width is as well due to scattering - the charge exchange reaction $\pi^{-} p \rightarrow \pi^{\circ} n$-and, therefore, is proportional to the square of $a^{-}$[36]. The Panofsky ratio $P=\Gamma\left(\pi^{-} p \rightarrow \pi^{\circ} n\right) / \Gamma\left(\pi^{-} p \rightarrow \gamma n\right)=1.546 \pm$ 0.009 37 accounts for the relative strength of radiative capture, the second channel contributing to the total broadening. For a recent analysis of $\pi \mathrm{H}$ atomic data see ref. 38].
It is known already from current algebra, that $a^{+}$vanishes in the chiral limit [9,10, acquiring a finite value only because of chiral symmetry breaking [12,39]. Hence, the s-wave $\pi N$ interaction is dominated by the isovector contribution with the consequence that it is difficult to extract $a^{+}$from pion-nucleon and nucleus data with $I \neq 0$.

Pionic deuterium $(\pi \mathrm{D})$ is of particular interest being the first natural choice to test the interaction of pions with isoscalar nuclear matter. Here, the leading order (LO) onebody contribution to the real part of the pion-deuteron scattering length $a_{\pi \mathrm{D}}$ depends only on the isoscalar quantity $a^{+}$. Considering the deuteron as a free proton and neutron plus corrections, one may write

$$
\begin{array}{rlrl}
\epsilon_{1 s}^{\pi \mathrm{D}} & \propto \operatorname{Re} a_{\pi \mathrm{D}} & \\
& =a_{\pi^{-} p \rightarrow \pi^{-} p}+a_{\pi^{-} n \rightarrow \pi^{-} n} & +\ldots \\
& = & 2 a^{+} & +\ldots
\end{array}
$$

Besides the corrections mentioned for the case of $\pi \mathrm{H}$, ellipses include here terms arising from multiple scattering, depending both on $a^{+}$and $a^{-}$, and absorptive contributions. Noteworthy, that the second order correction, dominated by $\left(a^{-}\right)^{2}$, exceeds even the magnitude of the leading order term because of $a^{+} \ll a^{-}$9, 10. Nuclear structure is taken into account by folding with the deuteron wave function [40,41,42]. The exact relation between $\epsilon_{1 s}^{\pi \mathrm{D}}$ and $\operatorname{Re} a_{\pi \mathrm{D}}$ is discussed in detail in sect. 3.1 .

Hence, the isopin scattering lengths are accessible from the shift measurements in $\pi \mathrm{H}$ and in $\pi \mathrm{D}$ already without ultimately precise data for the hadronic broadening in hydrogen being available 24. An effective constraint on $a^{+}$and $a^{-}$, however, is obtained when combining the triple $\epsilon_{1 s}^{\pi \mathrm{H}}, \Gamma_{1 s}^{\pi \mathrm{H}}$, and $\epsilon_{1 s}^{\pi \mathrm{D}}$. A complete set of data has been achieved for the first time during the last decade [43,44, 45, 46.

Such a constraint is highly desirable, because different methods exist to obtain the corrections needed for the extraction of the pure hadronic quantities $a^{+}$and $a^{-}$from $\pi \mathrm{H}$ and $\pi \mathrm{D}$ measurements. Recognising, among others, that corrections obtained using potential models were incomplete, significant effort went into calculations to establish an unambiguous relation between atomic data and $a^{+}$ and $a^{-}$, in particular within the framework of $\chi \mathrm{PT}$. It has been proven to be extendable in a defined way to 3-body interactions [47] and allows to treat electromagnetic and strong isopin-breaking terms on the same footing [5, 24, 48, 49, 50, 51,52,53,54,55,56,57,58,59. For a discussion and comparison of potential and EFT approaches see ref. [5].

As mentioned above, corrections for the $\pi N$ scattering lengths as obtained from $\pi \mathrm{H}$ amount to a few per cent of the leading contribution in complete contrast to the real part of the $\pi \mathrm{D}$ scattering length. Here, because of the smallness of $a^{+}$, the isospin-breaking contribution was found to be as large as $40 \%$ in NLO $\chi \mathrm{PT}[58$.

Finally, it turned out that for the extraction of the scattering lengths $a^{+}$from the atomic data within the framework of $\chi \mathrm{PT}$, the accuracy achievable is determined by one particular combination of LECs. It appears both in the correction term for $\epsilon_{1 s}^{\pi \mathrm{H}}$ and $\epsilon_{1 s}^{\pi \mathrm{D}}$, and thus constitutes a limit from the theoretical side [60]. In the case of $\Gamma_{1 \mathrm{~s}}^{\pi \mathrm{H}}$ 
the LECs involved are known better 5,61. Therefore, here the uncertainty of $a^{-}$is dominated by the experimental accuracy.

Being different from the $\pi \mathrm{H}$ case, the hadronic broadening in $\pi \mathrm{D}$ and, consequently, the imaginary part of the $a_{\pi \mathrm{D}}$ is not related to $\pi N$ scattering lengths $a^{+}$and $a^{-}$but to the strength of s-wave pion absorption. Open channels in negative pion absorption in deuterium at threshold are

$$
\begin{aligned}
\pi^{-} d & \rightarrow n n \\
& \rightarrow n n \gamma \\
& \rightarrow n n e^{+} e^{-} \\
& \rightarrow n n \pi^{0} .
\end{aligned}
$$

True absorption (5) represents the inverse reaction of pion production in nucleon-nucleon collisions, i.e., $\operatorname{Im} a_{\pi \mathrm{D}}$ is also a measure of the strength of s-wave pion production at threshold. For that reason, pion-production cross sections were used to estimate the $\pi \mathrm{D}$ level width before it was experimentally accessible 62. First attempts have been made to calculate the production strength rigorously also within the framework of $\chi \mathrm{PT}[63,64$ to be compared with the precise atomic data.

The relative strength of the dominant channels true absorption and radiative capture (6) was measured to be $S=\Gamma\left(\pi^{-} d \rightarrow n n\right) / \Gamma\left(\pi^{-} d \rightarrow n n \gamma\right)=2.83 \pm 0.0465$. The branching ratio of internal pair conversion (7) was determined to be $0.7 \% 66$. Charge exchange 8 is parity forbidden from s states and, therefore, only odd partial waves contribute which results in a fraction as small as $(1.45 \pm 0.19) \cdot 10^{-4} 67$. The corresponding partial $3 p$ level width can be neglected here. Hence, the relative strength of true absorption to all other final states contributing to the $1 s$ level width is found from the measured branching ratios to be

$$
S^{\prime}=\frac{\Gamma(n n)}{\Gamma(n n \gamma)+\Gamma\left(n n e^{+} e^{-}\right)}=2.76 \pm 0.04,
$$

i.e., about $2 / 3$ of the hadronic width is related to the pion production/absorption process $N N \leftrightarrow \pi N N$. The exact relation between pion-production strength and $\operatorname{Im} a_{\pi \mathrm{D}}$ is derived in sect. 3.2 .

The aim of this experiment is to provide data on the $\pi \mathrm{D}$ hadronic shift and width at least at about the level of accuracy achieved in recent or envisaged in ongoing theoretical calculations. A precise value for the shift will provide, together with the forthcoming new precision data for $\pi \mathrm{H} 68,69$, improved constraints for the $\pi N$ scattering lengths $a^{+}$and $a^{-}$. As a first result of this experiment, the s-wave pion production strength derived from the width has been outlined recently [70].

\section{Atomic cascade}

After pion capture in hydrogen isotopes, a quantum cascade starts from main quantum numbers $n \approx 16$ [71,72] (Fig.11). The upper and medium part of the atomic cascade is dominated by the collisional processes inducing
Stark mixing, Coulomb de-excitation, and ionization of neighbouring atoms (external Auger effect). In the lower part, X-ray emission becomes more and more important. In light atoms, only electric-dipole transitions contribute preferring steps with maximal $\Delta n$. The cascade time, estimated to be of the order of $0.1 \mathrm{~ns}$ for densities around 10 bar 73 , is much shorter than the pion's life time. In addition, molecular formation $\pi \mathrm{D}+\mathrm{D}_{2} \rightarrow[(d d \pi) d]$ ee must be considered. The influence of the above-mentioned processes is discussed in the following.

Radiative de-excitation is the only process where collisional effects on the line energy and width can be neglected. Doppler broadening from thermal motion is of the order of $10 \mathrm{meV}$. Pressure broadening amounts at maximum to $0.2 \mathrm{meV}$ for the densities and temperatures used in this experiment.

Stark mixing essentially determines the X-ray yields in exotic hydrogen. Because these systems are electrically neutral and small on the atomic scale, they penetrate surrounding atoms and, thus, experience a strong Coulomb field. Non-vanishing matrix elements $\left\langle n l m|\mathbf{E}| n l^{\prime} m^{\prime}\right\rangle$ in the presence of the electric field mix atomic states of the same principle quantum number $n$ according to the selection rules $\Delta l= \pm 1$ and $\Delta m=0$ [74. An induced $s$ state, from where pions disappear by nuclear reactions, leads to a depletion of the X-ray cascade. As the Stark mixing rate is proportional to the number of collisions during the exotic atom's life time, it explains the strong decrease of the X-ray yields with target density 73 . The yield of the $\pi \mathrm{D}(3 p-1 s)$ X-ray transition has been measured at tar-

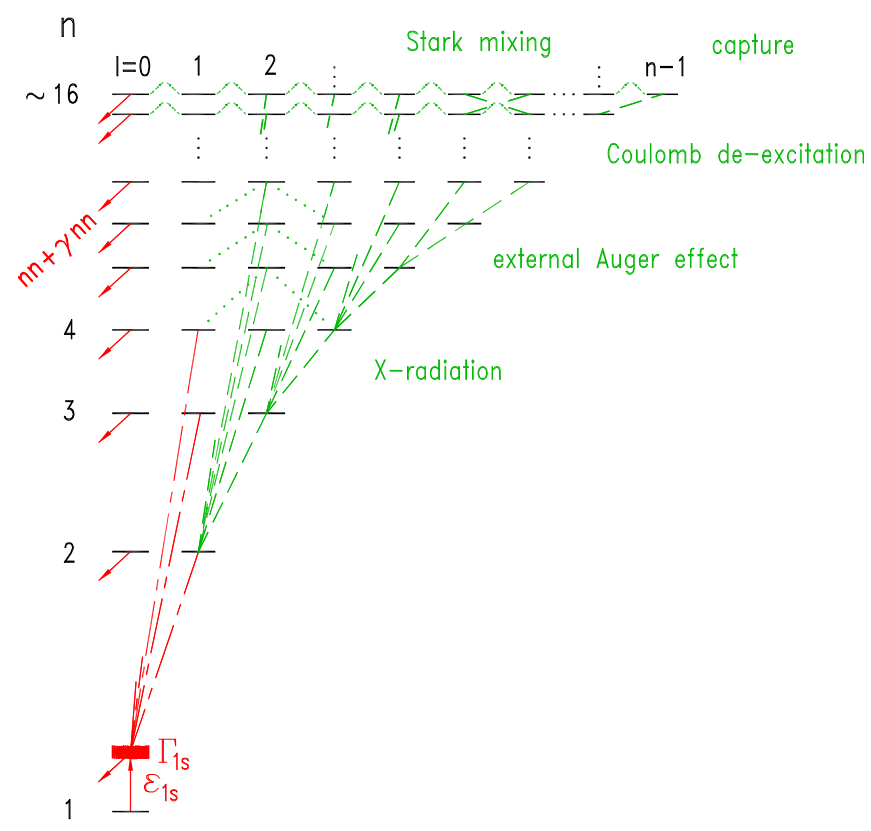

Fig. 1. De-excitation cascade in pionic deuterium. The energy of the X-ray emitted in the $\pi \mathrm{D}(3 p-1 s)$ transitions is $3.075 \mathrm{keV}$. Hadronic shift $\epsilon_{1 s}$ and width $\Gamma_{1 s}$ are of the order of 2.5 and $1 \mathrm{eV}$, respectively. The hadronic shift is defined as $\epsilon_{1 s} \equiv \mathrm{E}_{\exp }-\mathrm{E}_{\mathrm{QED}}$, i.e., a negative sign as is the case for $\pi \mathrm{D}$ corresponds to a repulsive interaction. 
get densities equivalent to 15 bar and 40 bar STP to be $(3.28 \pm 0.43) \%$ and $(1.72 \pm 0.16) \%$, respectively 75$]$.

The natural line width of the atomic ground state transition $(3 p-1 s)$ of about $1 \mathrm{eV}$ is dominated by the life time of the $1 s$ state. Nuclear reactions from the $3 p$ level contribute with less than $1 \mu \mathrm{eV}$ and the $3 p$-level radiative width amounts to $28 \mu \mathrm{eV}$ only. An estimate for the induced width from Stark mixing $(3 p \leftrightarrow 3 s)$ and external Auger effect at the $3 p$ state, based on transition rates for $\pi \mathrm{H}$ given in ref. [76, yields also negligibly small contributions of $\leq 1 \mu \mathrm{eV}$ at the target densities considered here. With a hadronic shift in $\pi \mathrm{D}$ being a factor of about 3 smaller than in $\pi \mathrm{H}$, Stark mixing might be enhanced, but strong effects are excluded because similar $\mathrm{K}$ yields have been observed for hydrogen and deuterium [75].

However, significant broadenings of the X-ray line induced by other cascade processes must be considered, in particular in the case of Coulomb de-excitation 79 . Here, the energy release during the transition $\left(n \rightarrow n^{\prime}\right)$ is converted into kinetic energy of the collision partners being the excited $\pi \mathrm{D}$ system and, predominantly, one atom of an $\mathrm{D}_{2}$ molecule 76,77,78. Such transitions dominate deexcitation from the start of the quantum cascade down to $n \approx 10$ and have been found to contribute even at lowest densities [80].

In lower-lying transitions, a significant energy gain occurs leading to a substantial Doppler broadening of subsequent X-ray transitions. The Doppler effect was directly observed in $\pi \mathrm{H}$ in time-of-flight spectra of monoenergetic neutrons from the charge exchange reaction at rest $\pi^{-} p \rightarrow$ $\pi^{0} n$ 81,82 and in an additional broadening of the line width of the muonic hydrogen $(3 p-1 s)$ X-ray transition 83 . The broadening turned out to be a superposition of several Doppler contributions attributed to different deexcitation steps $n \rightarrow n^{\prime}$ of the initial $\mu^{-} p$ system. Only $\Delta n=1$ transitions could be identified. A similar behaviour was found for pionic hydrogen. Here, a significant increase of the total line width is observed with decreasing $n$ of the initial state, which is attributed to the increasing energy gain of preceding Coulomb de-excitation steps 69, 84.

Acceleration due to Coulomb de-excitation is counteracted by elastic and inelastic scattering. This leads to a continuum of velocities below the ones well defined by a specific transition. Cascade calculations have been extended to include the development of the velocity dependence during de-excitation and, therefore, are able to predict kinetic energy distributions at the time of X-ray emission from a specific level (extended standard cascade model ESCM [76,77,78]). At present, calculations have been performed only for $\pi \mathrm{H}$ 76, 77, 78, 85, 86, 87. Figure2 shows such an ESCM prediction for the $3 p$ state.

In the case of $\pi \mathrm{D}$, the energies for the $\Delta n=1$ Coulomb de-excitation transitions $(7-6),(6-5),(5-4)$, and $(4-3)$, are at $12,20,38$, and $81 \mathrm{eV}$, respectively. Though kinetic energies differ only little from the $\pi \mathrm{H}$ case, velocities are significantly smaller (Fig.2) possibly changing the balance between acceleration and slowing down.

In the analysis of a measurement of the $\mu \mathrm{H}(3 p-1 s)$ line shape it turned out 83 , that first ESCM predictions are hardly able to describe the measured line width. Therefore, a model independent approach will be used to extract the relative strength of Doppler contributions directly from the X-ray data, which was applied successfully first in the neutron time-of-flight 82 and also in the $\mu \mathrm{H}(3 p-1 s)$ analysis. Here, the continuous kinetic energy distribution is replaced by a few narrow intervals at the energies, where significant contributions are expected. Positions and intensities of such contributions are then found by comparing a Monte-Carlo generated line shape to the data by means of a $\chi^{2}$ analysis. An approximation of the kinetic energy distribution by two narrow intervals, as used in the analysis of this experiment, is illustrated in fig.2. A detailed description is given in sect.5.2.

Auger transitions prefer de-excitation steps as small as possible, given that the energy gain exceeds the binding of the electron. Auger emission contributes mainly in the range $\Delta n \approx 6-10$ with $\Delta n=1$ transitions, but rapidly decreases for smaller $n$ [7, 77, 78. Because of the small recoil energies, Auger emission cannot contribute to the high energetic part of the kinetic energy distribution.

From muon-catalysed fusion experiments it is known that during $\mu \mathrm{H}+\mathrm{H}_{2}$ collisions metastable hybrid molecules are formed like $[(p p \mu) p] e e[88,89]$. An analogous process

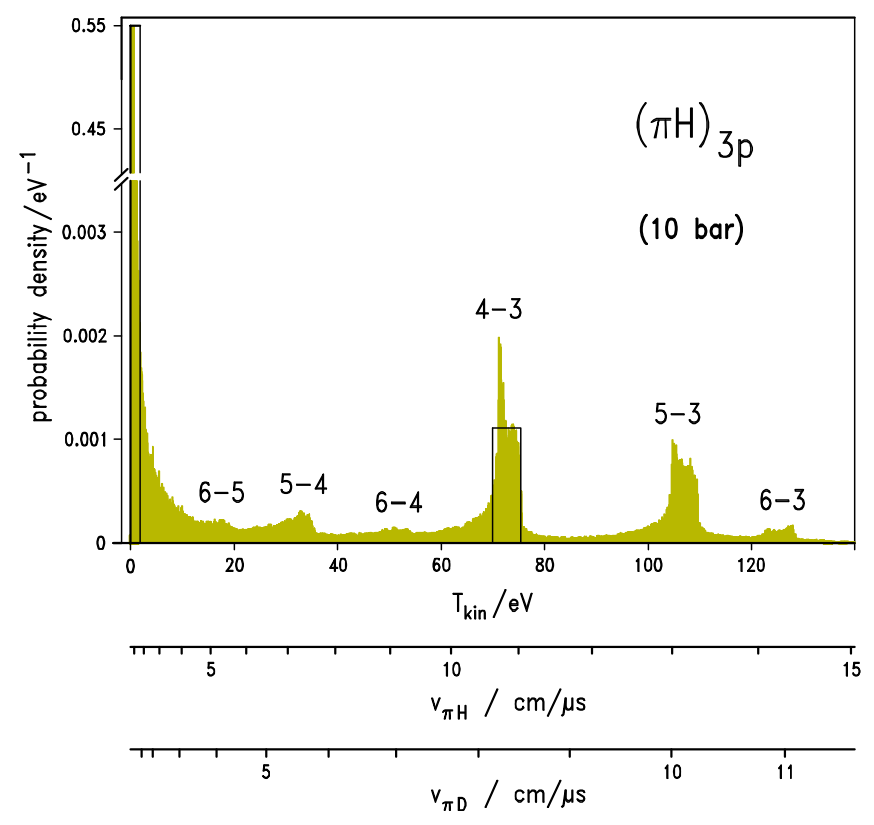

Fig. 2. Prediction for the kinetic energy distribution of the $\pi \mathrm{H}$ atom at the instant of $(3 p-1 s)$ transition for a density equivalent to 10 bar gas pressure together with the velocity scale. The velocities for the heavier $\pi \mathrm{D}$ system owing to the corresponding energy gains are given on the bottom. For the analysis of this $\pi \mathrm{D}$ experiment, the energy distribution, after scaling the energies from the $\pi \mathrm{H}$ to the $\pi \mathrm{D}$ case, was tentatively approximated by two narrow intervals representing a low-energy component $(0-2 \mathrm{eV})$ and a second one for the high-energy component stemming from the (4-3) Coulomb de-excitation transition (for details see sect. 5.2 . The low-energy component dominates the distribution (note the broken vertical scale). 
is expected in $\pi \mathrm{D}+\mathrm{D}_{2}$ collisions 89 . Evidence that resonant molecular formation takes also place from excited states has been found in muonic hydrogen [90.

Such complex' are assumed to stabilise non radiatively by Auger emission. Possible X-ray transitions from weakly bound molecular states before stabilisation would falsify the value for the hadronic shift determined from the measured X-ray energy due to satellite lines shifted to lower energies, whereas Auger stabilised molecules emit X-rays of at least $30 \mathrm{eV}$ energy less than the undisturbed transition 92 and are easily resolved in this experiment. Radiative decay out of such molecular states has been discussed and its probability is predicted to increase with atomic mass [91,92]. A weak evidence for an Auger stabilised transition is discussed in sect.6.1

As molecular formation is collision induced, the fraction of formed complex molecules and the X-ray rate should depend on the target density. Hence, a measurement of the $\pi \mathrm{D}(3 p-1 s)$ X-ray energy was performed at different densities to identify such radiative contributions (see sect.5.1.

\section{Strong interaction}

In this section, the relations are introduced which will be used to extract the complex pion-deuteron scattering length $a_{\pi \mathrm{D}}$ (sect.6.5) and the threshold parameter for s-wave pion production (sect. 6.6) from the measured hadronic level shift and broadening.

\subsection{Scattering length}

The hadronic pion-nucleus scattering length $a_{\pi \mathrm{A}}$ is related in leading order (LO) to the $n s$ level shifts $\epsilon_{n s}$ and widths $\Gamma_{n s}$ by the Deser-Goldberger-Baumann-Thirring (DGBT) formula [1]

$$
\epsilon_{n s}-i \frac{\Gamma_{n s}}{2}=-\frac{2 \alpha^{3} \mu^{2} c^{4}}{\hbar c} \cdot Z^{3} \cdot \frac{a_{\pi \mathrm{A}}^{\mathrm{LO}}}{n^{3}},
$$

with $\mu$ being the reduced mass of the bound system of pion and nucleus $A(Z, N)$. In eqs. 110 - 13 $\alpha$ denotes the fine structure constant.

Shift and width are measured using a Coulomb bound state. Therefore, the complex quantity $a_{\pi \mathrm{A}}^{\mathrm{LO}}$ as extracted from eq. 10 is not identical with the pure hadronic scattering length $a_{\pi \mathrm{A}}$. Usually, the Coulomb interaction is taken into account by Trueman's formula 93, 94, 95. which expresses the correction by an expansion in the ratio scattering length-to-Bohr radius. In the case of hydrogen $(Z=$ $1)$, the complete expansion can be written up to order $O\left(\alpha^{4}\right)$ in a compact form [5,33] yielding for the $1 s$ state of pionic deuterium 60 .

$$
\begin{aligned}
\epsilon_{1 s}-i \frac{\Gamma_{1 s}}{2}= & -\frac{2 \alpha^{3} \mu^{2} c^{4}}{\hbar c} a_{\pi \mathrm{D}} \\
& \cdot\left[1-\frac{2 \alpha \mu c^{2}}{\hbar c}(\ln \alpha-1) \cdot a_{\pi \mathrm{D}}+\delta_{\mathrm{D}}^{v a c}\right]
\end{aligned}
$$

The term $\delta_{\mathrm{D}}^{v a c}=0.51 \%$ accounts for the interference of vacuum polarisation and strong interaction [96]. Its uncertainty is assumed to be negligibly small compared to the experimental accuracy [5].

In the case of $\pi \mathrm{D}$, the total correction to $a_{\pi \mathrm{D}}$ is only about $1 \%$ (see eqs. 24) and (25)). Hence, eq. (11) is solved with sufficient accuracy by inserting the leading order result for $a_{\pi \mathrm{D}}^{\mathrm{LO}}$ as obtained from eq. 10 and the influence of the experimental uncertainty must not be considered here.

Real and imaginary part of the pure hadronic $\pi \mathrm{D}$ scattering length are then given by

$$
\begin{aligned}
\operatorname{Re} a_{\pi \mathrm{D}}= & -\frac{\hbar c}{2 \alpha^{3} \mu^{2} c^{4}} \cdot \epsilon_{1 s} \\
& \cdot \frac{1}{1-\frac{2 \alpha \mu c^{2}}{\hbar c}(\ln \alpha-1) \cdot \frac{\left(\operatorname{Re} a_{\pi \mathrm{D}}^{\mathrm{LO}}\right)^{2}-\left(\operatorname{Im} a_{\pi \mathrm{D}}^{\mathrm{LO}}\right)^{2}}{\operatorname{Re} a_{\pi \mathrm{D}}^{\mathrm{LO}}}+\delta_{\mathrm{D}}^{v a c}} \\
\operatorname{Im} a_{\pi \mathrm{D}}= & -\frac{\hbar c}{2 \alpha^{3} \mu^{2} c^{4}} \cdot \frac{\Gamma_{1 s}}{2} \\
& \cdot \frac{1}{1-\frac{2 \alpha \mu c^{2}}{\hbar c}(\ln \alpha-1) \cdot 2 \operatorname{Re} a_{\pi \mathrm{D}}^{\mathrm{LO}}+\delta_{\mathrm{D}}^{v a c}} .
\end{aligned}
$$

\subsection{Threshold parameter $\alpha$ and pion production}

The cross section for the reaction $p p \rightarrow \pi^{+} d$ is parametrised at low energies by 97

$$
\sigma_{p p \rightarrow \pi^{+d}}=\alpha C_{0}^{2} \eta+\beta C_{1}^{2} \eta^{3}+\ldots,
$$

where $\eta=p_{\pi}^{*} / m_{\pi}$ is the reduced momentum of the pion in the $\pi d$ rest frame. Here, $\alpha$ denotes the threshold parameter representing pure s-wave pion production. Approaching threshold, $\eta \rightarrow 0$, higher partial waves $(\beta, \ldots)$ vanish. A value for $\alpha$ is obtained by fits to the quantity $\sigma_{p p \rightarrow \pi^{+} d} / \eta$ and then extrapolated to zero energy. In particular at low energy, the correction factors $C_{i}$ which take into account the Coulomb interaction are an important source of uncertainty in the determination. For example, the leading order correction $C_{0}^{2}$ has been calculated to be as large as $30 \%$ with the additional difficulty to obtain an accurate error estimate 98, 99.

A second experimental approach to $\alpha$ is to exploit the $\pi \mathrm{D}$ ground state broadening, where uncertainties stemming from Coulomb correction factors and normalisation of cross sections are avoided. In order to derive in a model independent way the relation between $\alpha$ and $\operatorname{Im} a_{\pi \mathrm{D}}$ as obtained from pionic deuterium, purely hadronic (nonmeasurable) cross sections $\tilde{\sigma}$ are introduced to circumvent the problem of diverging Coulomb cross section at threshold. In this case $C_{i} \equiv 1$, and $\alpha$ and $\beta$ are pure hadronic quantities calculable, e.g., in the framework of $\chi \mathrm{PT}$. The hadronic production cross section then reads

$$
\tilde{\sigma}_{p p \rightarrow \pi^{+} d}=\alpha \eta+\beta \eta^{3}+\ldots .
$$

In addition, the reaction $n p \rightarrow \pi^{0} d$ can be used because in the limit of charge independence $2 \cdot \sigma_{n p \rightarrow \pi^{0} d}=\sigma_{p p \rightarrow \pi^{+} d}$. Restricting to pion-deuteron $s$ waves, in both processes the 
same transition of a nucleon pair ${ }^{3} P_{1}(I=1) \rightarrow{ }^{3} S_{1}(I=$ $0)$ occurs, where true pion absorption $\pi d \rightarrow n n$ induces the inverse reaction ${ }^{3} S_{1}\left[{ }^{3} D_{1}\right](I=0) \rightarrow{ }^{3} P_{1}(I=1)$ on the deuteron's isospin 0 nucleon-nucleon pair.

Detailed balance relates pion production and absorption by

$$
\tilde{\sigma}_{\pi^{+} d \rightarrow p p}=\frac{2}{3} \cdot\left(\frac{p_{p}^{*}}{p_{\pi}^{*}}\right)^{2} \cdot \tilde{\sigma}_{p p \rightarrow \pi^{+d}}
$$

with $p_{p}^{*}$ and $p_{\pi}^{*}$ being final state centre-of-mass (CMS) momenta [100. Neglecting Coulomb and isospin breaking corrections, charge symmetry requires for the transitions $\pi^{-} d \rightarrow n n$ and $\pi^{+} d \rightarrow p p$

$$
\left|M_{\pi^{-} d \rightarrow n n}\right|=\left|M_{\pi^{+} d \rightarrow p p}\right| .
$$

Isospin breaking effects are expected to be at most 1$2 \%$ 101,102.

A small difference in the transition rate results from the slightly larger phase space of the $\pi^{+} d \rightarrow p p$ reaction.

$$
\frac{\tilde{\sigma}_{\pi^{-} d \rightarrow n n}}{\tilde{\sigma}_{\pi^{+} d \rightarrow p p}}=\frac{p_{n}^{*}}{p_{p}^{*}}=\sqrt{\frac{\lambda\left(s, m_{n}^{2}, m_{n}^{2}\right)}{\lambda\left(s, m_{p}^{2}, m_{p}^{2}\right)}}=0.982,
$$

The CMS momenta of proton and neutron, expressed in invariant variables using the triangle function $\lambda$, are given by $p_{p, n}^{*}=\lambda^{1 / 2}\left(s, m_{p, n}^{2}, m_{p, n}^{2}\right) / 2 \sqrt{s}$ [103]. The value corresponds to $\pi^{ \pm} d$ at threshold, where the total CMS energy squared is $s=\left(m_{d}+m_{\pi^{ \pm}}\right)^{2}$. The atomic binding energy of the $\pi^{-} \mathrm{D}$ system is neglected.

Combining optical theorem, charge invariance, detailed balance and inserting the parametrisation of the $p p \rightarrow$ $\pi^{+} d$ cross section 15 , the imaginary part of the $\pi^{-} d \rightarrow$ $n n$ scattering length reads in terms of the $\pi^{+}$production threshold parameter $\alpha$

$$
\begin{aligned}
\operatorname{Im} a_{\pi^{-} d \rightarrow n n} & =\frac{p_{\pi}^{*}}{4 \pi} \cdot \tilde{\sigma}_{\pi^{-} d \rightarrow n n} \\
& =\frac{p_{\pi}^{*}}{4 \pi} \cdot \tilde{\sigma}_{\pi^{+} d \rightarrow p p} \cdot\left(\frac{p_{n}^{*}}{p_{p}^{*}}\right) \\
& =\frac{p_{\pi}^{*}}{4 \pi} \cdot \frac{2}{3} \cdot\left(\frac{p_{p}^{*}}{p_{\pi}^{*}}\right)^{2} \cdot \tilde{\sigma}_{p p \rightarrow \pi^{+} d} \cdot\left(\frac{p_{n}^{*}}{p_{p}^{*}}\right) \\
& =\frac{1}{6 \pi} \cdot \frac{\left(p_{p}^{*} \cdot p_{n}^{*}\right)}{m_{\pi}} \cdot \alpha .
\end{aligned}
$$

To relate $\operatorname{Im} a_{\pi^{-} d \rightarrow n n}$ given by pion production to the imaginary part of the pion-deuteron scattering length $\operatorname{Im} a_{\pi D}$ as obtained from the atomic system $\pi \mathrm{D}$, a correction must be applied for the non true absorption channels. Taking into account the relative strength $S^{\prime}$ of true absorption to other s-state processes (9) and exploiting $\lambda(x, y, y)=x(x-4 y) 103$, one obtains

$$
\begin{aligned}
\operatorname{Im} a_{\pi \mathrm{D}} & =\left(1+\frac{1}{S^{\prime}}\right) \cdot \operatorname{Im} a_{\pi^{-} d \rightarrow n n} \\
& =\left(1+\frac{1}{S^{\prime}}\right) \cdot \frac{\sqrt{\left(s-4 m_{p}^{2}\right)\left(s-4 m_{n}^{2}\right)}}{24 \pi m_{\pi}} \cdot \alpha \\
& =2.48 \cdot 10^{-5} m_{\pi}^{-1} \mu b^{-1} \cdot \alpha .
\end{aligned}
$$

\section{Experimental setup}

The experiment was performed at the $\pi$ E5 channel of the proton accelerator at PSI, which provides a low-energy pion beam with intensities of up to a few $10^{8} / \mathrm{s}$ (Fig. 3). Pions of $112 \mathrm{MeV} / \mathrm{c}$ were injected into the cyclotron trap II 104, 105 and decelerated by using a set of degraders optimised to the number of pion stops in a cylindrical cryogenic target cell of $22 \mathrm{~cm}$ length and $5 \mathrm{~cm}$ in diameter placed in the centre of the trap. Its magnetic field, perpendicular to the beam direction, guides the particles after a few turns towards the target.

The cell was filled with deuterium gas cooled by means of a cold finger to adjust the target density by temperature. Data were taken at three different conditions, which are equivalent to gas densities of $3.3,10$, and 17.5 bar at a temperature of $20^{\circ} \mathrm{C}$. The corresponding target parameters may be found in table 2. About $0.5 \%$ of the incoming pions are stopped per bar equivalent pressure of $\mathrm{D}_{2}$. Xradiation could exit the target cell axially through a $5 \mu \mathrm{m}$ thick mylar ${ }^{\circledR}$ window. The window foil is supported by $1 \mathrm{~mm}$ thick horizontal aluminum bars constructed with a $6 \mathrm{~mm}$ spacing.

X-rays from the $\pi \mathrm{D}(3 p-1 s)$ transition were measured with a Johann-type Bragg spectrometer equipped with a spherically bent Si crystal cut along the (111) plane and having a large radius of curvature of $R=2982.2 \pm 0.3 \mathrm{~mm}$ in order to minimise aberrations. The crystal plate was attached by molecular forces to a glass lens polished to optical quality, which defines the curvature. A possible miscut and its orientation were determined in a dedicated measurement [106]. The reflecting area of $10 \mathrm{~cm}$ in diam-

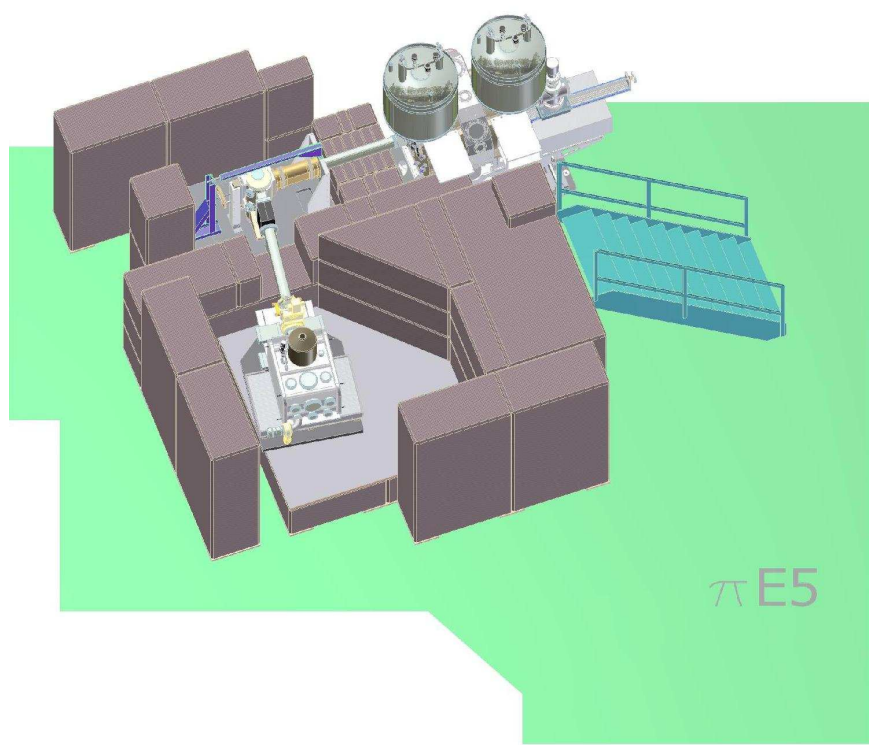

Fig. 3. Setup of the $\pi \mathrm{D}$ experiment in the $\pi \mathrm{E} 5$ area at the Paul Scherrer Insitut (PSI). The roof of the concrete shielding is omitted to show the vacuum system connecting the cyclotron trap (upper right), crystal chamber (upper left) and the cryostat of the X-ray detector (bottom left). Details of the mechanical setup are shown in figs. 4 and 5 
eter was restricted by a circular aperture to $95 \mathrm{~mm}$ in diameter to avoid edge effects and to $60 \mathrm{~mm}$ horizontally in order to keep the Johann broadening small 107,108.

Such a spectrometer is able to measure simultaneously an energy interval according to the width of the X-ray source when using a correspondingly extended X-ray detector. Being pixel detectors, charge-coupled devices (CCDs) are ideal detectors for X-rays in the few $\mathrm{keV}$ range because they combine an intrinsic position resolution with the good energy resolution of semiconductor detectors. In this setup an array of $3 \times 2$ CCDs was used covering in total $72 \mathrm{~mm}$ in height and $48 \mathrm{~mm}$ in width 109 .

Monte Carlo studies show that about $2 / 3$ of the intensity of the reflection is covered by the height of the CCD array. The solid angle of the crystal with respect to the target is $\approx 6 \cdot 10^{-5}$ and the target fraction accepted by the crystal's angular width is $\approx 5 \cdot 10^{-3}$. Including window absorption and assuming a peak reflectivity of $40 \%$ for the Bragg crystal, the overall efficiency of the spectrometer results in about $10^{-7}$.

The CCDs are realised in open electrode architecture to minimise X-ray absorption in the surface structures and a depletion depth of about $30 \mu \mathrm{m}$ yields a quantum efficiency of about $80 \%$ in the $3-4 \mathrm{keV}$ range. The pixel size of $40 \mu \mathrm{m}$ provides a sufficient two-dimensional position resolution to measure precisely the shape of the diffraction image. The relative orientation of the individual CCDs as well as the pixel size at the operating temperature of $-100^{\circ} \mathrm{C}$ was obtained by means of an optical measurement using a nanometric grid 112 . The detector surface was oriented perpendicular to the direction crystal - detector.

In the Johann setup, the energy calibration must be provided by a reference line of known energy. The best

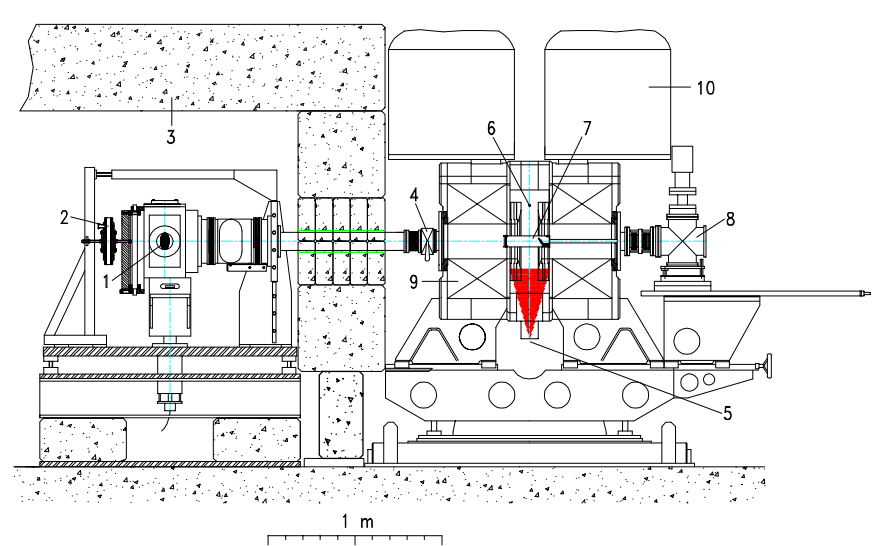

Fig. 4. Sectional drawing of the connection of the cyclotron trap to the crystal chamber of the spectrometer. The symmetry axis of the magnetic field coincides with the line center of the trap to center of the Bragg crystal. 1: Bragg crystal, 2: traction relaxation, 3: concrete shielding, 4: gate valve, 5: X-ray tube (emission cone indicated), 6: position of pion beam without $\mathrm{B}$ field, 7: cryogenic target cell including calibration target (right end), 8: target cell support and cryogenic generator, 9: magnet coils of cyclotron trap, 10: LHe dewars.
Table 1. Data to adjust the spectrometer in Johann setup. The Bragg angle $\Theta_{\mathrm{B}}$ is calculated using a lattice distance $d$ of $2 \mathrm{~d}_{111}=(0.62712016 \pm 0.00000001) \mathrm{nm}$ [110]. $n$ denotes the order of reflection. For the $\pi \mathrm{D}(3 p-1 s)$ energy, here the experimental result of ref. 44 is given. $y_{\mathrm{CD}}=R \cdot \sin \left(\Theta_{\mathrm{B}}+\Delta \Theta_{\mathrm{IRS}}\right)$ are calculated focal lengths taking into account the index of refraction shift $\Delta \Theta_{\text {IRS. The }}$ Ga K $\alpha$ energies are taken from the compilation of ref. [111.

\begin{tabular}{lccccc}
\hline & $\begin{array}{c}\text { energy } \\
/ \mathrm{eV}\end{array}$ & $n$ & $\Theta_{\mathrm{B}}$ & $\Delta \Theta_{\mathrm{IRS}}$ & $\begin{array}{c}y_{\mathrm{CD}} \\
/ \mathrm{mm}\end{array}$ \\
\hline $\mathrm{Ga} \mathrm{K} \alpha_{1}$ & $9251.674 \pm 0.066$ & 3 & $39^{\circ} 52^{\prime} 22.3^{\prime \prime}$ & $2.4^{\prime \prime}$ & 1912.42 \\
$\pi \mathrm{D}(3 p-1 s)$ & $3075.52 \pm 0.70$ & 1 & $40^{\circ} 0^{\prime} 11.7^{\prime \prime}$ & $21.8^{\prime \prime}$ & 1917.84 \\
$\mathrm{Ga} \mathrm{K} \alpha_{2}$ & $9224.484 \pm 0.027$ & 3 & $40^{\circ} 0^{\prime} 44.1^{\prime \prime}$ & $2.4 ”$ & 1917.98 \\
\hline
\end{tabular}

angular matching for the $\pi \mathrm{D}(3 p-1 s)$ measurement was found with the gallium $\mathrm{K} \alpha$ fluorescence lines. Here, the Ga $\mathrm{K} \alpha_{2}$ transition was chosen because of its smaller experimental error (Table1). The energy of the measurement line is obtained by the angular distance to the reference line, which is calculated from the position offset of the two reflections on the detector and the distance crystal detector.

The fluorescence target was made of a $25 \times 20 \mathrm{~mm}^{2}$ GaAs plate mounted in the rear part of the gas cell outside the pion stop volume and $82 \mathrm{~mm}$ off the centre of the cyclotron trap away from the crystal (Fig. 4). X-rays were excited by means of an X-ray tube mounted on a window of the cyclotron trap chamber below the gas cell.

In order to exclude the distortion of the $\pi \mathrm{D}(3 p-1 s)$ line shape from beam-induced excitation of Ga X-rays, data were taken using $\mathrm{H}_{2}$ gas at an equivalent density of

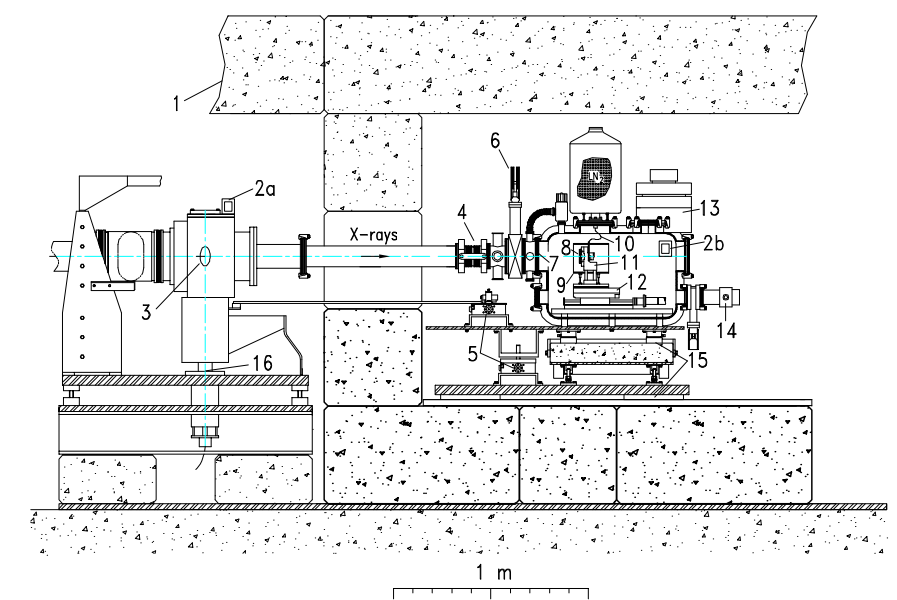

Fig. 5. Sectional drawing of the connection of the crystal chamber to the X-ray detector. 1: concrete shielding, 2a and 2b: inclination sensors, 3: Bragg crystal, 4: compensator, 5: linear tables for crystal and detector adjustment, 6: gate valve, 7: $5 \mu \mathrm{m}$ Mylar window, 8: CCD array, 9: cold trap, 10: copper braid, 11: cold finger, 12: translation table for focal length adjustment, 13: detector readout electronics, 14: turbomolecular pump, 15: air cushion support, 16: crystal chamber support. 
10 bar. Using a hydrogen filling guarantees similar stopping conditions for the pion beam. No Ga X-rays could be identified during a measuring time corresponding to about $15 \%$ of the one used for $\mathrm{D}_{2}$ at the same density. Other sources of background are discussed in sect.5.

The distance from the centre of the crystal to the centre of the cyclotron trap was $2100 \mathrm{~mm}$, about $10 \%$ outside the Rowland circle given by the focal condition $R \cdot \sin \Theta_{\mathrm{B}}$. The advantage of placing the $\mathrm{X}$-ray source somewhat off the focal position is an averaging over non uniformities of the target. Both GaAs and $\mathrm{D}_{2}$ target were extended enough that no cuts in the tails of the reflection occur. The distance crystal - detector (Fig. 5), chosen to be at the assumed $\pi \mathrm{D}$ focal length, was found to be $\mathrm{y}_{\mathrm{CD}}=1918.1 \pm 0.5 \mathrm{~mm}$ from a survey measurement.

Alternating measurements of the Ga fluorescence radiation and the $\pi \mathrm{D}$ line were performed at least once per day. The Ga fluorescence X-rays were in addition used to monitor the stability of the line position.

The spectrometer response was measured at the energies 3104,2765 , and $2430 \mathrm{eV}$ using the narrow M1 Xray lines from helium-like argon, chlorine, and sulphur produced in a dedicated electron-cyclotron ion resonance trap (ECRIT) 113 114, 115. It turned out that the resolution function at a given energy can be built up from the ideal response calculated from the dynamical theory of diffraction for a perfect flat crystal (intrinsic resolution) convoluted with the geometrical imaging at the measurement position by means of a Monte-Carlo ray-tracing code and by folding in an additional Gaussian contribution. The intrinsic resolution is calculated here with the code XOP [116]. The Gaussian models possible imperfections of the crystal material and mounting and was found to be sufficiently precise, that no difference is visible by eye between data and Monte-Carlo simulations.

The value for the Gaussian width at the energy of the $\pi \mathrm{D}(3 p-1 s)$ transition energy of $3075 \mathrm{eV}$ was found from the fit to the results for argon, chlorine, and sulphur to be $(122 \pm 8) \mathrm{meV}$. The narrow structure in fig. 8 - middle shows the Monte-Carlo generated response for the setup of the $\pi \mathrm{D}$ experiment. The total width of this resolution function is $(436 \pm 3) \mathrm{meV}$ (FWHM) and close to the theoretical limit of $403 \mathrm{meV}$ - calculated by means of XOP for the intrinsic resolution of a silicon crystal cut along the (111) plane.

Details on the experimental setup and analysis may be found elsewhere [117.

\section{Analysis}

Raw data of the X-ray detector consist of the digitized charge contents and a position index of the pixel. The granularity of the CCDs allows for efficient background rejection by means of pattern recognition (cluster analysis). Photoelectrons from few keV X-ray conversion are stopped within a few micrometer only and, therefore, deposit charge in one or two pixels with one common boundary. Beam induced background, mainly high energetic photons from neutrons produced in pion absorption and cap-

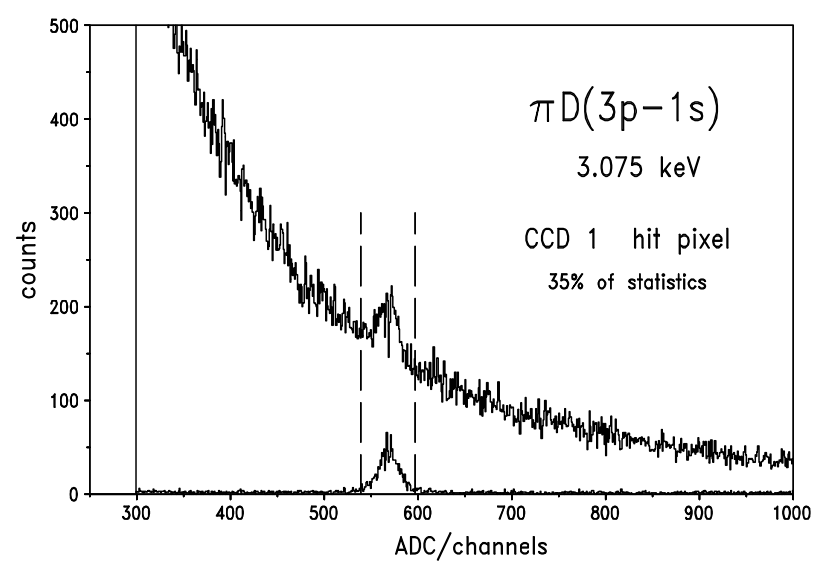

Fig. 6. Spectrum of the collected (digitized) charge in one CCD before and after cluster analysis of one of the 3 CCDs covered by the $\pi \mathrm{D}(3 p-1 s)$ reflection. Data are from the measurement at 10 bar equivalent density and correspond to $35 \%$ of the $\pi \mathrm{D}$ statistics collected with CCD 1 . The peak around ADC channel 570 is due to the transition energy of $3.075 \mathrm{keV}$. Vertical dashed lines indicate the applied "energy cut" of $\pm 2.5 \sigma$ allowing for further background reduction.

tured in surrounding nuclei, lead to larger structures. Together with a massive concrete shielding (Fig.3) such events are highly suppressed. Defect pixels are masked by software.

At first, the cluster analysis is performed. As expected only single $(\approx 75 \%)$ or two pixel events $(\approx 25 \%)$ contribute. The spectra of the collected charge cleaned in this way show a pronounced peak originating from the $3 \mathrm{keV} \pi \mathrm{D}$ X-rays (Fig.6). For each CCD an individual energy calibration was performed by using the $\pi \mathrm{D}(3 p-1 s)$ line, necessary because of the different gain and noise behaviour of the various devices. The second calibration point is zero because the noise peak is suppressed on-line during data acquisition which reduces the amount of data to be recorded substantially. The energy resolution in terms of charge is determined by means of a Gaussian fit and found to be between 170 and $300 \mathrm{eV}$ at $3 \mathrm{keV}$.

The good resolution in energy (i.e., in collected charge) by CCDs allows to apply a narrow "energy cut" in the ADC spectra (Fig.6). Thus an additional and significant background reduction is achieved in the two dimensional position spectra (Fig.7 - top). Assuming a Gaussian shape for the energy resolution and setting various windows ranging from $\pm 1 \sigma$ to $\pm 4 \sigma$, the influence was studied of the peak-to-background ratio on the result for the hadronic broadening and found to be marginal. The minimum statistical error is achieved for a cut of $\pm 2.5 \sigma$. The X-ray count rates at the equivalent densities of $3.3,10$, and 17.5 bar are given in table 2 .

The hit pattern of the X-rays on the CCD surface shows a curvature originating from the imaging properties of the crystal spectrometer. The curvature is corrected by means of a parabola fit before projecting onto the axis of dispersion, which is equivalent to an energy axis (Fig. 7). 
The penetration depths of the $\pi \mathrm{D}(3 p-1 s)$ and the Ga X-rays differ significantly (5 and $105 \mu \mathrm{m}$ for 3.075 and $9.224 \mathrm{keV}$, respectively 118 ). Ga K X-rays also convert at or beyond the boundary of the depletion region of the CCD, where charge diffusion is already significant. Therefore, in this case compact clusters up to size 9 were accepted. As the Ga calibration measurements were performed without pion beam in the experimental area, no background events occur and, hence, no suppression algorithm is necessary to clean the Ga spectra. For one Ga calibration measurement, typically $4000 \mathrm{Ga} \mathrm{K} \alpha_{2}$ events were recorded. The total line width was found to be $(2.9 \pm$ $0.1) \mathrm{eV}$, which is two standard deviations above the natural line width of $(2.66 \pm 0.13) \mathrm{eV}$ as given in ref. [119].
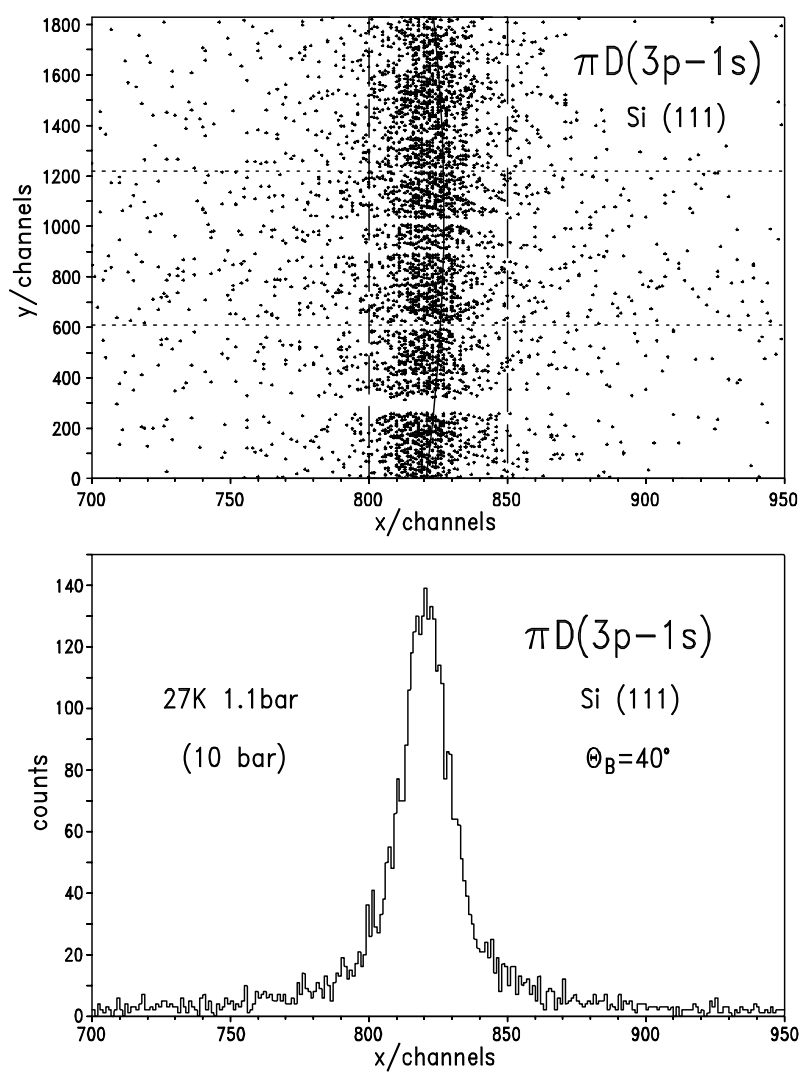

Fig. 7. Top - Scatter plot of the $\pi \mathrm{D}(3 p-1 s)$ reflection measured at a density equivalent to 10 bar (NTP). Its curvature is obtained by a parabola fit. Bottom - projection to the axis of dispersion after curvature correction. One channel (pixel) in the direction of dispersion $(x)$ corresponds in first order reflection to $76.402 \pm 0.001 \mathrm{meV}$.

\subsection{Energy calibration}

The tabulated values for the Ga K $\alpha$ energies [11] were obtained using the compound GaAs [120 - the same material used in this experiment, i.e., a possible chemical shift is irrelevant for this calibration. The position of the $\mathrm{Ga}$
Table 2. Experiment parameters. Rates (counts/Cb) are meant for a detector width of $14 \mathrm{~mm}$ and normalised to the integrated accelerator proton current (typically $2 \mathrm{~mA}$ ) for "energy cut" of $\pm 2.5 \sigma$. The equivalent density of the target gas is expressed as pressure value at a temperature of $20^{\circ} \mathrm{C}(\mathrm{NTP})$. The number of Ga calibration runs for each density is indicated in the last column. The backgound rate is density independent and scales linearly with the width of the "energy cut". The background given here stems from a detector area of $17.28 \mathrm{~cm}^{2}$ corresponding to $3 \mathrm{CCDs}$.

\begin{tabular}{|c|c|c|c|c|}
\hline \multirow{2}{*}{$\begin{array}{c}\mathrm{D}_{2} \text { density } \\
\text { (equivalent } \\
\text { / bar }\end{array}$} & \multirow{2}{*}{$\begin{array}{l}\text { target } \\
\mathrm{T} \\
/ \mathrm{K}\end{array}$} & \multirow{2}{*}{$\begin{array}{l}\text { parameters } \\
\text { pressure } \\
\text { / bar }\end{array}$} & $\begin{array}{c}\text { counts } \\
\pi \mathrm{D}(3 p-1 s)\end{array}$ & \multirow[t]{2}{*}{$\begin{array}{c}\text { no. of Ga } \\
\text { calibrations }\end{array}$} \\
\hline & & & total $/ \mathrm{Cb}$ & \\
\hline $3.3 \pm 0.1$ & 33 & 0.51 & $1448 \pm 491.98 \pm 0.07$ & 10 \\
\hline $10.0 \pm 0.3$ & 27 & 1.09 & $4010 \pm 746.40 \pm 0.12$ & 8 \\
\hline $17.5 \pm 0.4$ & 25 & 1.36 & $4877 \pm 807.35 \pm 0.12$ & 7 \\
\hline background & & & $1.64 \pm 0.11$ & \\
\hline
\end{tabular}

$\mathrm{K} \alpha_{2}$ line was determined applying a single Voigt profile in the fit, which is also the procedure used for the tabulated values 120 . The matching of the angular positions owing to the $\mathrm{Ga} \mathrm{K} \alpha_{2}$ and $\pi \mathrm{D}(3 p-1 s)$ energies is evident from fig. 8 .

The $\pi \mathrm{D}$ line was modeled both with a Voigt profile, where Doppler broadening and response function together are approximated by a single Gaussian, and the true response as determined from the ECRIT data convoluted with the imaging properties by means of a ray tracing MC code and including the Doppler contributions from Coulomb de-excitation. Both methods yield the same position value within a few hundreds of one CCD pixel.

Various corrections to the measured line positions must be applied. Significant are, besides the index of refraction shift, bending and penetration correction, a shift of the gallium line due to the non-uniform illumination of the fluorescence target, and mechanical shifts from temperature changes from the nitrogen filling of the detector. These corrections are discussed in the following and listed together with less significant corrections in table 3 .

- Index of refraction. The Ga calibration line and the $\pi \mathrm{D}(3 p-1 s)$ transition were measured in third and first order diffraction (table 1). As the index of refraction strongly depends on the X-ray wave length a significant correction must be applied in order to obtain the right Bragg angle difference. The index of refraction $\Delta \Theta_{\text {IRS }}$ is calculated here from the atomic scattering factors as used by the code XOP 116]. The accuracy of such amplitudes is claimed to be of the order of $1 \%$ for 3 and $9 \mathrm{keV}$, respectively, as are the angular corrections derived from them. The amplitudes of various sets vary by less than $0.5 \%$ 121, 122 .

- Crystal bending. Crystal bending leads to a depth dependence of the lattice spacing $d$, where close to the surface the maximum lattice distance is assumed. Because of the difference in energy and accordingly the different mean penetration depth of the X-rays, the average lattice spacing and, consequently, the diffrac- 

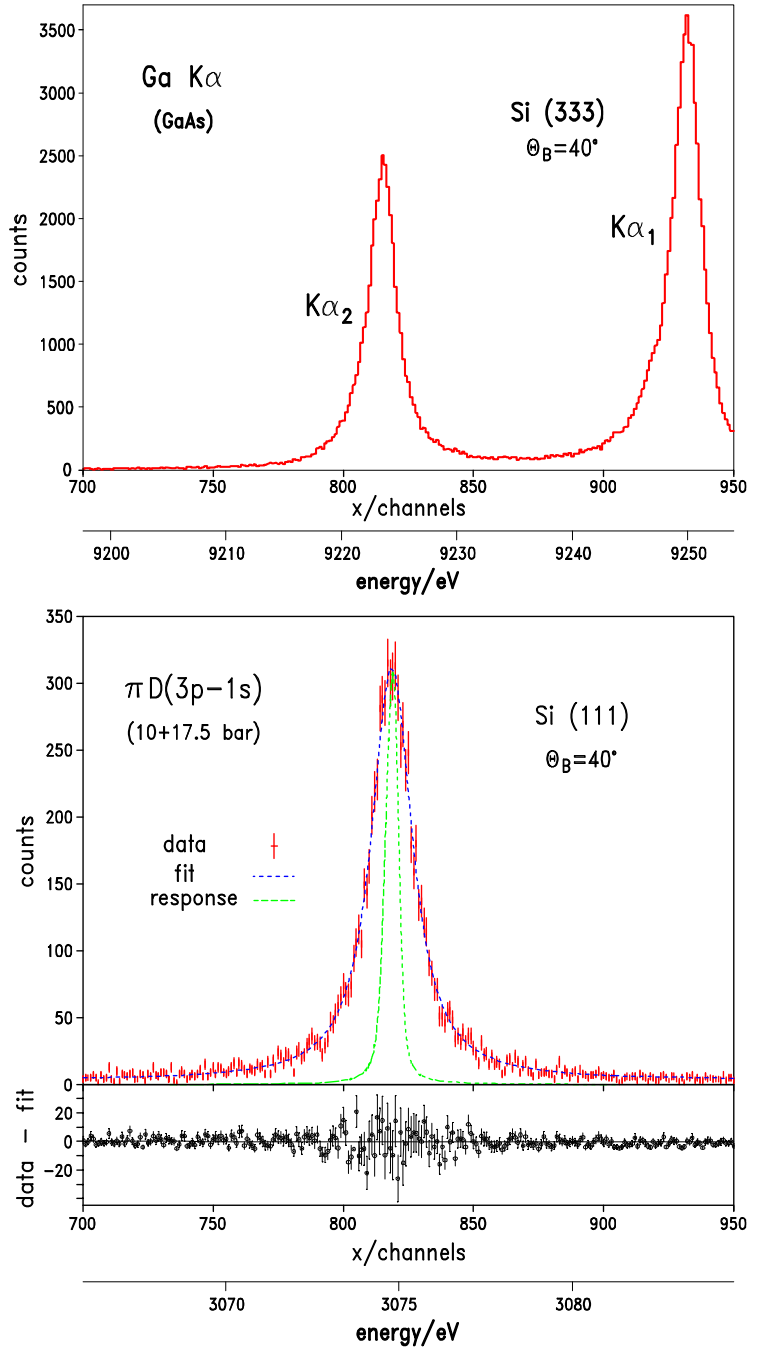

Fig. 8. Top - Ga $\mathrm{K} \alpha_{2}$ calibration line. The $\mathrm{K} \alpha_{1}$ component is slightly reduced by the asymmetric emission characteristics of the X-ray tube. Middle - sum spectrum of the $\pi \mathrm{D}(3 p-$ $1 s$ ) transition measured at the equivalent densities of 10 and 17.5 bar. The narrow structure represents the response function of the crystal spectrometer (normalised to the height of the measured spectrum). Bottom - residuum of data and fit. The fit is performed without assuming any Doppler components.

tion angle is different. The change of $d$ depends on the elastic properties of the nonisotropic crystal material and is calculated following the approach of ref. [123 using the value $\nu^{\prime}=0.182$ for Poisson's ratio together with an expression for the angular correction as given by ref. 124 .

- Penetration depth. The difference in penetration depth into the crystal leads to an offset of higher energetic X-rays to smaller diffraction angles, which has been corrected according to the approach of ref. [124. The penetration depths (including absorption) were obtained from the code XOP to $0.72 \mu \mathrm{m}$ and $3.84 \mu \mathrm{m}$ for 3.075 and $9.225 \mathrm{keV}$, respectively.
- Fluorescence-target illumination. The intensity profile of the X-ray tube, used to illuminate the GaAs target, was found to decrease in the direction of dispersion towards larger Bragg angles. Therefore, the centre of gravity of the line is shifted towards smaller Bragg angles or higher energies. The shape of the intensity distribution has been measured precisely and used for a Monte Carlo simulation to quantify the shift.

- Mechanical stability. Due to a fixed sequence for refilling the X-ray detector with liquid nitrogen (twice per day) and the gallium calibration (once per day after filling), small mechanical distortions arose from the cold nitrogen gas. These distortions were monitored every $15 \mathrm{~min}$ with two inclination sensors (of $1 \mu \mathrm{rad}$ precison), one attached to the crystal chamber and the second to the detector cryostat. In this way, both short and long term movements of the vertical crystal axis relative to the X-ray detector were recognized. For each of the three measurement periods an average correction was determined from the inclinometer data. The maximal deviations were found to be about $\pm 0.1 \mathrm{mrad}$ corresponding to about one pixel.

- Curvature correction. The curvature of the reflection is determined from a parabola fit both to the $\pi \mathrm{D}$ and the $\mathrm{Ga} \mathrm{K} \alpha_{2}$ line, which can be assumed to be equal within the envisaged accuracy. The centres of gravity are determined choosing a left and right boundary aligned with the curvature of the reflection itself, the reflection divided vertically in 12 slices per CCD, and iterated until the minimal $\chi^{2}$ is reached. The maximum position by the various choices for the line and the boundaries are summarised in the given uncertainty.

- Defocussing. The small difference in the focal length of the $\pi \mathrm{D}$ and the $\mathrm{Ga} \mathrm{K} \alpha_{2}$ leads to a small defocussing correction, which was determined by means of a Monte-Carlo simulation.

- Focal length. The parts constituting the mechanical connection between crystal and detector were adjusted to the assumed focal length of the $\pi \mathrm{D}(3 p-1 s)$ transition. Their length and the non parallelity of the flanges were precisely surveyed resulting in an uncertainty of the distance of $\pm 0.5 \mathrm{~mm}$.

- Alignment. A possible deviation from the detector surface from being perpendicular to the direction crystal centre to detector centre leads to a correction for the measured distance of the $\pi \mathrm{D}$ and $\mathrm{Ga} \mathrm{K} \alpha_{2}$ reflection. The uncertainty is composed of the setup of the CCD array inside the cryostat and the deviation from being parallel of the flanges of the connecting tubes, which were obtained from a survey measurement.

- Height alignment. If X-ray source, bragg crystal and detector centre are aligned perfectly in-plane, a symmetric reflection with respect to this plane occurs at the detector surface. A misalignment causes a tilt of the reflection inducing a small error using the parabola approach for the curvature correction. The vertical positions of target, crystal, and detector centre relative to the incoming pion beam were measured to be 206.5, 205.1 , and $206.1 \mathrm{~mm}$ with an accuracy of $\pm 0.2 \mathrm{~mm}$. 
- Pixel size. The average pixel size of the CCDs was determined in a separate experiment to be $(39.9775 \pm$ $0.0006) \mu \mathrm{m}$ at the operating temperature of $-100^{\circ} \mathrm{C}$ 112 .

- Temperature normalisation. The crystal temperature was monitored regularly during the measurements. Though of minor impact, the lattice constant tabulated for $22^{\circ} \mathrm{C}$, was readjusted to the average temperature of $30^{\circ} \mathrm{C}$. The temperature was found to be stable within less than $\pm 1^{\circ} \mathrm{C}$. Hence, any position variation due to the change of the lattice constant can be neglected.

As described above, the total uncertainty in the determination of the $\pi \mathrm{D}(3 p-1 s)$ transition energy is dominated by the one of the Ga calibration line $( \pm 27 \mathrm{meV})$. The systematic error of about $6 \mathrm{meV}$ is about $50 \%$ of the statistical uncertainty (sect.6.1) and comparable to the uncertainty of the calculated electromagnetic transition energy (sect.6.2).

\subsection{Line width}

For comparison with data, the line shape is constructed by folding the response function (see sect.4 and fig. 8p with the Doppler induced width derived from a given kinetic energy distribution (e.g., as shown in fig.2), and a Lorentzian representing the natural width. Based on the experience of the study of the $\mu \mathrm{H}(3 p-1 s)$ line shape 83 , in a cascade model free approach, the kinetic energy distribution is approximated by narrow intervals of a few $\mathrm{eV}$ width at energies which are inspired by the energy release of Coulomb de-excitation transitions. Such an energy spectrum assuming only a low-energy $(0-2 \mathrm{eV})$ and one high-energy component (owing to the $(4-3)$ Coulomb de-excitation) is included in fig. 2.

Spectra constructed in this way are compared to data by means of a $\chi^{2}$ analysis using the MINUIT package 125 . Natural line width, total intensity of the line, background, and relative weight of the Doppler contributions are free parameters of the fit. The sum of the Doppler contributions is normalised to one.

Following again the approach from the analysis of the $\mu \mathrm{H}(3 p-1 s)$ transition, one tries to identify consecutively individual Doppler contributions by using first a single kinetic-energy component starting at energy zero and variable width. This corresponds to $\pi \mathrm{D}$ systems not being accelerated or already moderated down again by collisions to energies of a few eV. The $\chi^{2}$ analysis shows that the upper boundary of the low-energy contribution must not exceed $8 \mathrm{eV}$. This result was achieved independently for the spectra taken at 10 bar and the 17.5 bar equivalent density (Fig.9). The result for the natural line width $\Gamma_{1 s}$ turned out to be insensitive to the upper boundary when keeping its value at $8 \mathrm{eV}$ or below. Therefore, the lowenergy component was fixed to the interval $0-2 \mathrm{eV}$ in the further analysis.

The most important higher energetic components are expected at about 80 and $115 \mathrm{eV}$ stemming from the
Table 3. Angular corrections (in seconds of arc (")) with associated uncertainties as well as other sources of systematic and calibration errors occuring in the determination of the $\pi \mathrm{D}(3 p-1 s)$ transition energy and hadronic shift $\epsilon_{1 s}$. The total angular correction being the sum of the individual terms and associated uncertainties are converted to meV. The total systematic error owing to experiment and setup (errors are assumed to be uncorrelated) is given conservatively as the quadratic sum and is calculated assuming on average the mechanical stability of the 17.5 bar measurement. For comparison, the statistical error and the uncertainty of the QED transition energy (see sects.6.1 and 6.2 is shown together with the one of the Ga calibration line.

\begin{tabular}{|c|c|c|c|c|c|}
\hline \multirow{2}{*}{$\begin{array}{l}\text { uncertainty } \\
\text { correction } \\
\text { index of refraction }\end{array}$} & \multirow{2}{*}{$\begin{array}{l}\mathrm{D} \\
/ " \\
-\end{array}$} & \multirow{2}{*}{$\begin{array}{c}\mathrm{Ga} \mathrm{K} \alpha \\
/ " \\
2.4\end{array}$} & \multicolumn{3}{|c|}{$\begin{array}{c}\text { total correction } \\
/ \mathrm{meV}\end{array}$} \\
\hline & & & -42.6 & \pm & 0.4 \\
\hline index of refraction & 21.8 & - & 387.2 & \pm & 3.9 \\
\hline crystal bending & -3.73 & -3.65 & -1.4 & $\stackrel{+}{+}$ & $\begin{array}{l}0.4 \\
0.9\end{array}$ \\
\hline penetration depth & -0.06 & -0.32 & 4.6 & $\stackrel{+}{-}$ & $\begin{array}{l}1.1 \\
2.7\end{array}$ \\
\hline illumination GaAs target & 0 & -1.075 & 19.1 & \pm & 3.8 \\
\hline mechanical stability 3.3 bar & 0.24 & & 4.2 & \pm & 2.5 \\
\hline mechanical stability 10 bar & 0.71 & & 12.6 & \pm & 4.0 \\
\hline mechanical stability 17.5 bar & 0.80 & & 14.2 & \pm & 1.3 \\
\hline curvature correction & & & & \pm & 2.0 \\
\hline defocussing & 0 & -0.006 & 0.11 & \pm & 0.01 \\
\hline focal length & & & & \pm & 0.05 \\
\hline alignment crystal-detector & & & & $\begin{array}{l}+ \\
-\end{array}$ & $\begin{array}{c}0 \\
0.015\end{array}$ \\
\hline height alignment & & & & $\stackrel{+}{-}$ & $\begin{array}{c}0 \\
0.016\end{array}$ \\
\hline pixel size & & & & \pm & 0.14 \\
\hline temperature renormalisation & & & & \pm & 0.02 \\
\hline bias due to asymmetric errors & & & -0.4 & & \\
\hline total systematic error & & & & $\begin{array}{l}+ \\
- \\
\end{array}$ & $\begin{array}{l}6.0 \\
6.6 \\
\end{array}$ \\
\hline statistical error & & & & & 10.9 \\
\hline $\mathrm{E}_{\mathrm{QED}}$ & & & & \pm & 7.9 \\
\hline $\mathrm{Ga} \mathrm{K} \alpha_{2}$ & & & & \pm & 27 \\
\hline
\end{tabular}

Coulomb de-excitation transitions $(4-3)$ and $(5-3)$. Searches for any of these contributions failed, even when using the sum spectrum of the two measurements at 10 and 17.5 bar. Varying energy or width of the high-energy Doppler contribution did not change this result.

In this respect, pionic deuterium differs significantly from pionic and muonic hydrogen. There, such high-energy contributions were clearly identified from the width analysis of the X-ray transitions [46, 69, 83]. For that reason, the ESCM calculation of the kinetic energy distribution for the $\pi \mathrm{H}(3 p-1 s)$ case, scaled to $\pi \mathrm{D}$ kinematics (Fig.2), is unable to reproduce the $\pi \mathrm{D}(3 p-1 s)$ line shape, because it contains rather strong contributions from $(4-3)$ and 


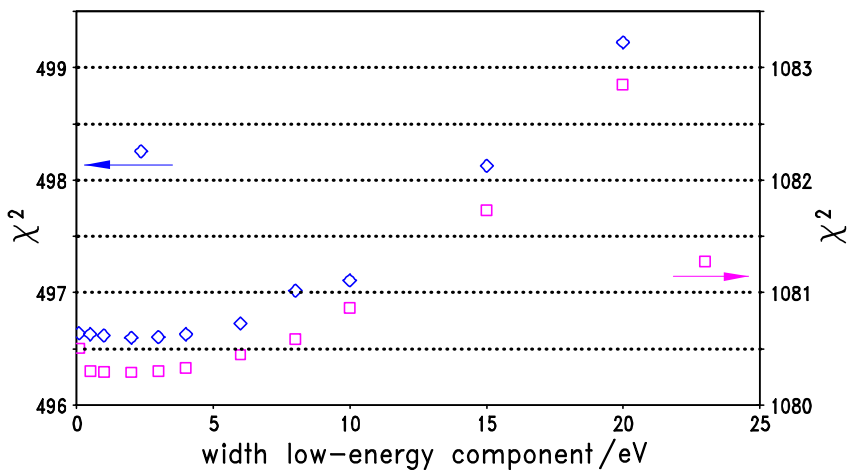

Fig. 9. Search for evidence of an extended low-energy component in the kinetic energy distribution by including in the fit of the data a uniform kinetic energy distribution of variable width starting at $T_{\text {kin }}=0$. Allowing for kinetic energies above about $8 \mathrm{eV}$ increasingly downgrades the quality of the fit. Diamonds (left $\chi^{2}$ scale) are due to the 10 bar data. Squares (right scale) are from a combined fit to the two spectra taken at 10 and 17.5 bar. Degrees of freedom in the fits are 487 (left scale) and 974 (right).

$(5-3)$ Coulomb de-excitation. At present, no explanation has been found for such an unequal behaviour.

It has been studied in detail which fraction of highenergy components may be missed in the fit by studying Monte-Carlo generated spectra for the statistics collected for the sum of the 10 and 17.5 bar measurements. The intensity of the Doppler contributions was not restricted to positive values to allow an unbiased search for the minimum $\chi^{2}$ (Fig. 10 - top). The normalization to one is then maintained by a correspondingly increased value for the other component.

The probability to miss a Doppler component, stemming from kinetic energies around $80 \mathrm{eV}$ and corresponding to the (4-3) transition, is displayed in fig. 10 - bottom as a function of its relative strength. It can be seen, that a contribution of $25 \%$ or larger can hardly be missed. For $10 \%$ relative intensity, the chance is about $15 \%$ to find intensities $\leq 0$ in the fit. Taking symmetric limits around the maximum at about $10 \%$ relative frequency (Fig. 10 - top) corresponds to $1 \sigma$ with respect to the full distribution. For each set of conditions 400 simulations were performed.

Assuming no Doppler components yields an upper limit for $\Gamma_{1 s}$ which is identical to the result obtained when using only the low-energy component of $0-2 \mathrm{eV}$. The residuum of such a fit is shown in fig. 8 - bottom. The limit of sensitivity for a component at $80 \mathrm{eV}$ kinetic energy of $10 \%$ yields a lower bound for $\Gamma_{1 s}\left(-\triangle \Gamma_{\text {sys }}\right)$ corresponding to the above-mentioned $1 \sigma$ criterium (Fig. 10). The distribution of the results for the weight of this component and the Lorentz width $\Gamma$ reflects the fluctuations owing to the limited statistics (Fig.11).

From the various above-mentioned sets, each with 400 simulations, a possible systematic deviation (bias) of the analysis code was examined. Such a deviation stems from an imperfect description of the probability distribution by the fit model 126, here used to extract $\Gamma_{1 s}$, which becomes more and more important with decreasing statistics. Where for the individual spectra measured at 3.3, 10, and 17.5 bar the bias was found to depend significantly on the statistics $((-38 \pm 3),(17 \pm 2)$, and $(11 \pm 2) \mathrm{meV})$, for the sum spectrum $(10+17.5)$ bar it becomes almost negligible $((-2 \pm 2) \mathrm{meV})$. The error of $\approx 2 \mathrm{meV}$ of the bias is given by the average of the results from the 400 Monte-Carlo spectra, which in turn determines the number of simulations necessary.

The behaviour of the bias is understood as follows. For lower statistics, the suppression of the tails results in smaller widths, whereas in the case of higher statistics the fit tends to include background into the tail. The background has been found to be constant in all spectra. The systematical uncertainty of $\Gamma_{1 s}$ due to different background levels within the limits as obtained from the fit to the data is estimated to below $1 \mathrm{meV}$ by means of Monte Carlo simulations.

Again 400 Monte-Carlo generated spectra, based on a calculated ESCM energy distribution, were used to quantify the sensitivity to any further high-energy component.
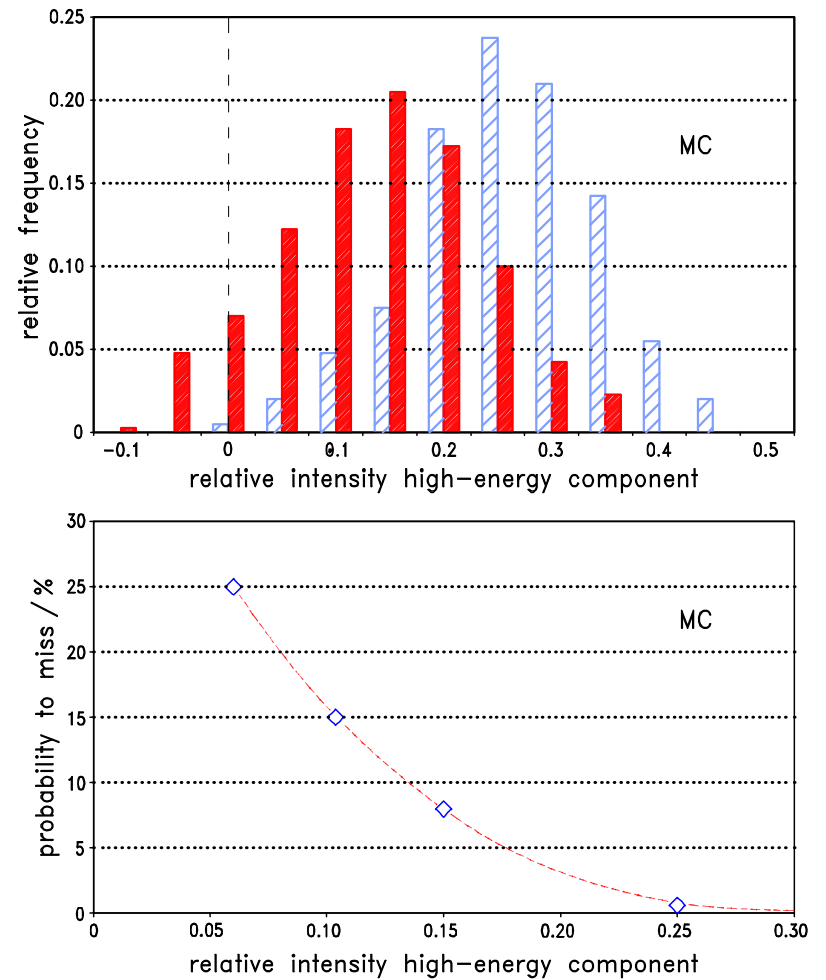

Fig. 10. Top - distributions of relative intensity of a Doppler broadened component induced by the $(4-3)$ Coulomb deexcitation transition found from fits to Monte-Carlo (MC) generated spectra. The relative strength used for the simulations is $10 \%$ (filled) and $25 \%$ (hatched) relative intensity, respectively. In both cases, the result is based on 400 spectra generated for the statistics of the sum of the 10 and 17.5 bar data. Bottom - probability to miss a Doppler contribution corresponding to the $(4-3)$ Coulomb de-excitation (modeled by a uniform kinetic energy distribution from $(76-84) \mathrm{eV}$ ) as a function of its relative intensity. 
An additional $6 \%$ fraction corresponding to the predicted strength of the $(5-3)$ de-excitation together with a $10 \%$ contribution from the $(4-3)$ is identified by the fit in more than $2 / 3$ of all cases. In the other $1 / 3$ of the fits, the $(5-3)$ fraction is absorbed into the $(4-3)$ contribution leading to an overestimate of its intensity and a shift to higher energies.

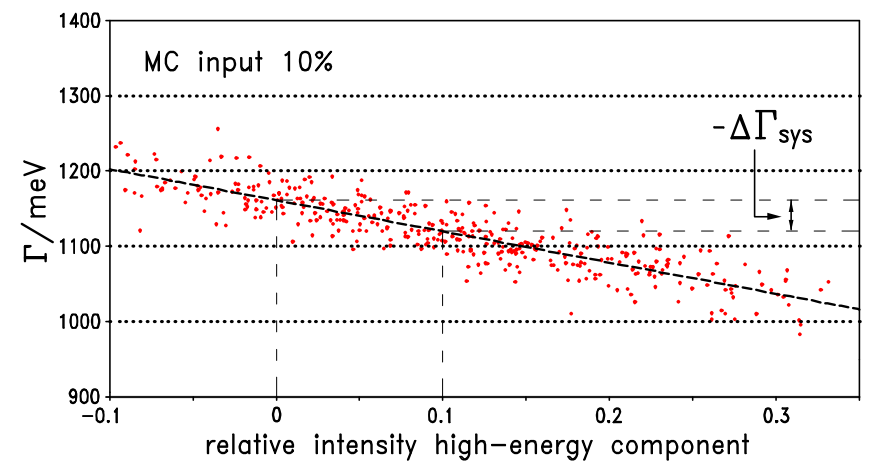

Fig. 11. Distributions of the Lorentz width $\Gamma$ versus the relative intensity of the Doppler broadened component stemming from the $(4-3)$ Coulomb de-excitation. The results are obtained from 400 Monte-Carlo simulations performed for the statistics of the sum of the 10 and 17.5 bar data. Input values are $10 \%$ relative intensity for the Doppler affected component and $1130 \mathrm{eV}$ for $\Gamma$.

No evidence for an additional, i.e., asymmetric broadening from non resolved satellites due to molecular formation has been found within the experimental accuracy. This is corroborated by the observation that a possible density dependence of the $\pi \mathrm{D}(3 p-1 s)$ energy contradicts the expected behaviour (see sects.6.1 and 6.3).

\section{Results and discussion}

\subsection{Experimental $\pi \mathbf{D}(3 p-1 s)$ transition energy}

The results for the $\pi \mathrm{D}(3 p-1 s)$ X-ray energy are summarized in table 4 taking already into account the corrections and their uncertainties as given in table 3 . The values obtained for the three different target densities are consistent within two standard deviations (neglecting the common uncertainty of the $\mathrm{Ga} \mathrm{K} \alpha_{2}$ energy).

It is concluded that we cannot identify radiative deexcitation from molecular states within the experimental accuracy, even more, as it is expected that such a process decreases the $\pi \mathrm{D}$ transition energy with density. Noteworthy, that also the analysis of the $\mu \mathrm{H}(3 p-1 s)$ line shape yields no evidence for a broadening owing to molecular effects 83 .

Inspecting the background at the low-energy side of the $\pi \mathrm{D}$ transition for possible Auger stabilised molecules, a weak structure was found separated by $(28.6 \pm 0.5) \mathrm{eV}$. Its relative intensity was determined to be $(3.4 \pm 1.6) \cdot 10^{-3}$ of the main line.
A weighted average is calculated for the $\pi \mathrm{D}(3 p-1 s)$ transition energy from the results for the three different target densities by using the individual contributions to the statistical and systematical error. The final value and error (quadratic sum) is obtained when combining these errors with the uncertainty of $\Delta \mathrm{E}_{\mathrm{Ga}}$ for the $\mathrm{GaK} \alpha_{2}$ line:

$$
\mathrm{E}_{\pi \mathrm{D}(3 p-1 s)}=(3075.583 \pm 0.030) \mathrm{eV} .
$$

The experimental uncertainty is dominated by the error of the $\mathrm{Ga} \mathrm{K} \alpha_{2}$ calibration line. In case a significant theoretical progress requires further improvement, the calibration error could be reduced by a factor of about 2 in a dedicated measurement using a double flat crystal spectrometer.

Table 4. Measured $\pi \mathrm{D}(3 p-1 s)$ transition energy and associated uncertainties.

\begin{tabular}{ccccc}
\hline $\begin{array}{c}\text { equivalent } \\
\text { density } \\
\text { /bar }\end{array}$ & $\mathrm{E}(3 p-1 s)$ & \multicolumn{4}{c}{$\Delta \mathrm{E}(3 p-1 s)$} \\
& $/ \mathrm{eV}$ & stat & sys & $\Delta \mathrm{E}_{\mathrm{Ga}}$ \\
\hline 3.3 & 3075.509 & \pm 28 & +6 & \pm 27 \\
10 & 3075.594 & \pm 17 & +7 & \pm 27 \\
17.5 & 3075.599 & \pm 16 & +6 & \pm 27 \\
\hline weighted average & 3075.583 & \pm 11 & \pm 7 & \pm 27 \\
\hline
\end{tabular}

\subsection{Electromagnetic $\pi \mathbf{D}(3 p-1 s)$ transition energy}

In order to extract the hadronic shift, the pure electromagnetic transition energy has been recalculated within the framework of quantum electrodynamics (QED). The contributions to the level energies are given in table 5 The error of the calculated QED transition energy $\mathrm{E}_{\mathrm{QED}}$ $=3077.939 \pm 0.008 \mathrm{eV}$ is dominated by the uncertainty of the charged pion mass [127] $( \pm 7.72 \mathrm{meV})$, whereas the ones of the charge radii of the deuteron $( \pm 1.19 \mathrm{meV})$ and the pion [127] $( \pm 0.95 \mathrm{meV})$ contribute only marginal. The numerical accuracy is better than $1 \mathrm{meV}$. For this calculation, a deuteron charge radius $r_{\mathrm{D}}=(2.12809 \pm 0.00031) \mathrm{fm}$ has been used as derived from the most recent value of the proton charge radius 128 and the radii difference obtained from the hydrogen-deuterium isotope shift measured by means of two-photon spectroscopy [129.

The correction to the ground-state energy arising from the electric polarizability of the deuteron of $(-26.3 \pm 0.5)$ $\mathrm{meV}$ is obtained by scaling from a calculation for muonic deuterium 130,131. Contributions from the polarizability of the pion 132 are three orders of magnitude smaller and, consequently, are neglected.

The strong-interaction shift of the $\pi \mathrm{D} 3 p$ state is estimated as for the s-wave in leading order by the sum of the pion-proton and pion-neutron interaction. The $\pi d$ scattering volume may be expressed by twice the isoscalar and angular momentum averaged parameter $c_{0}$ of the Kisslinger potential 3, 48, 62, which can be calculated from the $\pi N$ 
Table 5. Contributions to the pure electromagnetic binding and the $(3 p-1 s)$ transition energies. The terms vac. pol. 11, 13, and 21 stand for Uehling, Wichmann-Kroll, and Källén-Sabry contributions. The shift value due to the polarizability of the deuteron is derived from results given in refs. 130,131. A "0" indicates a negligibly small but finite value.

\begin{tabular}{lccc}
\hline & $3 p$ & $1 s$ & $\mathrm{E}_{\mathrm{QED}}(3 p-1 s)$ \\
& $/ \mathrm{eV}$ & $/ \mathrm{eV}$ & $/ \mathrm{eV}$ \\
\hline Coulomb & -384.31079 & -3458.52422 & 3074.21344 \\
self-energy + finite size & 0 & 0.00220 & -0.00220 \\
vac. pol. 11 & -0.01432 & -3.72948 & 3.71516 \\
muon vac. pol. 11 & 0 & -0.00034 & -0.00034 \\
vac. pol. 13 & 0 & 0.00002 & -0.00002 \\
vac. pol. 21 & -0.00013 & -0.02793 & 0.02780 \\
recoil 1 & -0.00004 & -0.00297 & 0.00293 \\
relativistic recoil & 0 & 0.00029 & -0.00029 \\
two-loop vac. pol. & 0 & -0.00577 & 0.00577 \\
pion finite size & 0 & -0.04764 & 0.04764 \\
deuteron polarizability & 0 & -0.0263 & 0.0263 \\
$\epsilon_{3 p}$ & -0.000008 & - & 0.000008 \\
atom recoil & - & - & -0.00235 \\
\hline total & -384.32528 & -3462.26684 & 3077.93922 \\
\hline
\end{tabular}

scattering volumes. Values are given, e.g., in ref. [133. Inserting the result for $2 \cdot c_{0}$ in the Trueman formula for the $3 p$ state [93 94, one obtains a negligibly small shift of $\epsilon_{3 p} \approx 8 \mu \mathrm{eV}$.

\subsection{Strong-interaction shift $\epsilon_{1 s}$}

Subtracting measured and calculated QED transition energies, the hadronic shift is obtained to

$$
\epsilon_{1 s}=-(2356 \pm 31) \mathrm{meV}
$$

The negative sign indicates a repulsive interaction, i.e., the strong interaction reduces the atomic $1 \mathrm{~s}$ level binding energy.

Our result is compared to previous measurements in table 7 and fig. 12 . Note that the electromagnetic transition energy $\mathrm{E}_{\mathrm{QED}}$ differs slightly in the analysis of the various experiments. Worth mentioning, that the results of the three precision experiments are in good agreement though three different calibration lines have been used.

Combining the results of this experiment and the ones given in refs. 44 and 45, yields a weighted average of $\epsilon_{1 s}=(-2379 \pm 19) \mathrm{meV}$ assuming any absence of a density dependence for the $\pi \mathrm{D}(3 p-1 s)$ transition energy.

Inspecting fig. 12 suggests that an extrapolation to density zero yields the value aimed at. A linear fit yields a slope of $(6.3 \pm 1.7) \mathrm{meV} / \mathrm{bar}$ and $\epsilon_{1 \mathrm{~s}}=(2445 \pm 27) \mathrm{meV}$ at density zero. However, at present there is no explanation for such a density dependence (see sect.2), which might be subject for a next generation experiment and, therefore, we stick to the density independent average given in 22 .

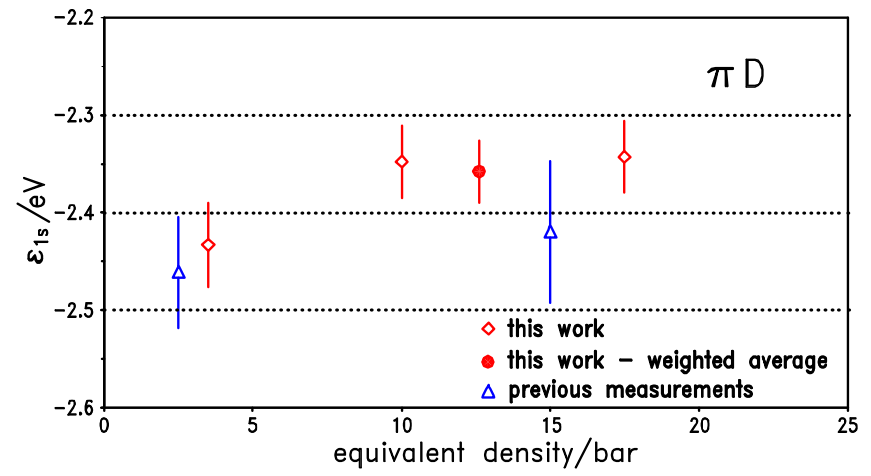

Fig. 12. Experimental information on the hadronic shift in pionic deuterium. The error of the individual measurements at $3.3,10$, and 17.5 bar equivalent density and, therefore, also of the weighted average is dominated by the uncertainty of the $\mathrm{Ga} \mathrm{K} \alpha_{2}$ energy. The results from the two previous experiments at 2.5 bar [45] and 15 bar 44] are adjusted according to the new values for the pure electromagnetic transition energies (Table7).

\subsection{Strong-interaction width $\Gamma_{1 s}$}

Corresponding to the discussion in sect.5.2, the hadronic broadening is extracted by introducing only a low-energy component from $0-2 \mathrm{eV}$ in the kinetic energy distribution. The combined result is obtained by averaging according to the statistical weight (Table6). The large negative error is dominated by the uncertainty that a Doppler contribution of the level of about $10 \%$ might be missed in the analysis.

Combining all errors (quadratic sum), we obtain

$$
\Gamma_{1 s}=\left(\begin{array}{r}
1171+23 \\
-49
\end{array}\right) \mathrm{meV}
$$

The result is in good agreement with the earlier measurements, but a factor of about 3 more precise (Table 7 and fig. 13). The weighted average of the results of this experiment, 44], and [45] is $\Gamma_{1 s}=\left(1165_{-38}^{+22}\right) \mathrm{meV}$.

Table 6. Pionic deuterium ground state width $\Gamma_{1 s}$ as obtained from the individual measurements at $3.3,10$, and 17.5 bar and the sum spectrum at $(10+17.5)$ bar. The weighted average is calculated from the result of the sum spectrum $(10+17.5)$ bar

\begin{tabular}{|c|c|c|c|}
\hline \multirow{2}{*}{$\begin{array}{c}\text { equivalent } \\
\text { density } \\
\text { / bar }\end{array}$} & \multirow{2}{*}{$\begin{array}{c}\Gamma_{1 s} \\
/ \mathrm{meV}\end{array}$} & \multicolumn{2}{|c|}{$\Delta \Gamma_{1 s}$} \\
\hline & & $\begin{array}{l}\text { stat } \\
/ \mathrm{meV}\end{array}$ & $\begin{array}{l}\text { sys } \\
\text { / meV }\end{array}$ \\
\hline 3.3 & 1246 & \pm 71 & $\begin{array}{l}+0 \\
+152\end{array}$ \\
\hline 10 & 1177 & \pm 38 & $\begin{array}{l}+0 \\
+92\end{array}$ \\
\hline 17.5 & 1121 & \pm 32 & $\begin{array}{l}+0 \\
-81\end{array}$ \\
\hline$(10+17.5)$ & 1162 & \pm 24 & $\begin{array}{l}+0 \\
-\quad 43 \\
\end{array}$ \\
\hline weighted average & 1171 & \pm 23 & $\begin{array}{l}+0 \\
-43\end{array}$ \\
\hline
\end{tabular}
and the one of the 3.3 bar measurement. 
Table 7. Transition energies and hadronic effects found for pionic deuterium and corresponding experimental conditions. The total error of the measured transition energy $\mathrm{E}_{\exp }$ is calculated quadratically from the statistical and systematical contributions. The hadronic shift is defined by $\epsilon_{1 s} \equiv \mathrm{E}_{\exp }-\mathrm{E}_{\mathrm{QED}}$. Note the change of $\mathrm{E}_{\mathrm{QED}}\left(^{*}\right)$ due to a new calculation performed for the analysis of this experiment which shifts the result of [44] to $\epsilon_{1 s}=-2419 \pm 100 \mathrm{meV}$. Also for the $(2 p-1 s)$ transition a new QED value of $2597.519 \pm 0.008 \mathrm{eV}$ is obtained resulting in $\epsilon_{1 s}=-2461 \pm 55 \mathrm{meV}$ for the experiment of [45]. No uncertainties for the QED values have been reported for the experiments decribed in refs. [45, 135, 136].

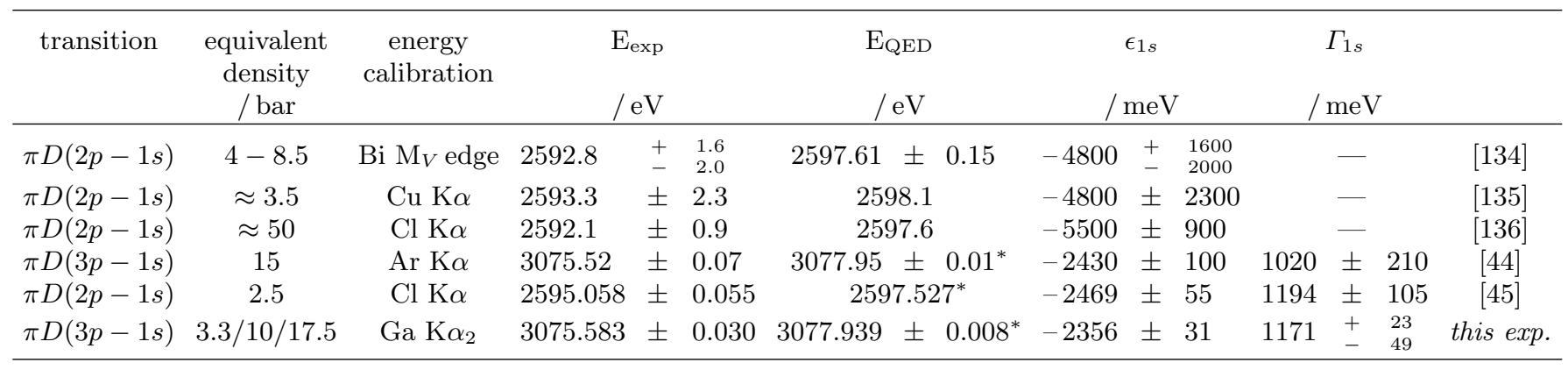

Again, a possible density dependence might be suspected (Fig 13). Performing a linear fit-taking into account the asymmetry of the errors-yields $\Gamma_{1 s}=(1229 \pm$ $75) \mathrm{meV}$ at density zero and a slope of $(-6.8 \pm 4.8) \mathrm{meV} /$ bar. Also here, no reason was found for such a density dependence and we keep the density independent result.

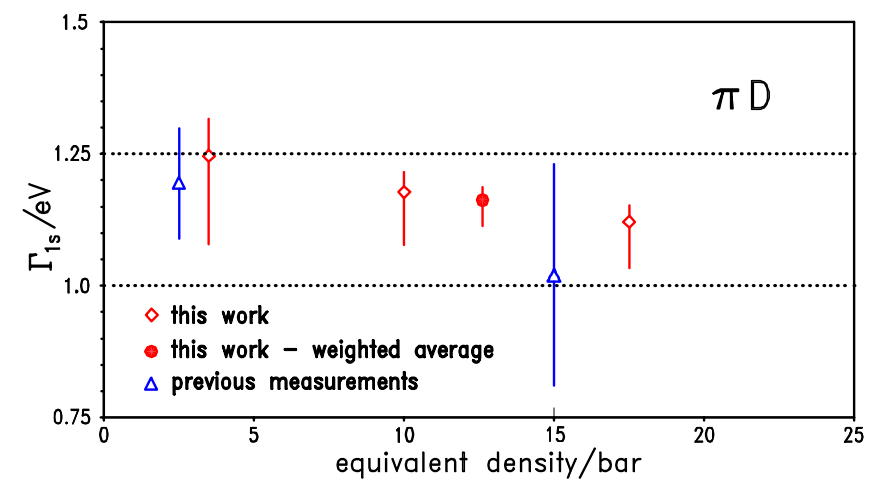

Fig. 13. Experimental information on the hadronic broadening in pionic deuterium. Previous measurements were performed at 2.5 bar 45] and 15 bar 44 equivalent density.

\subsection{Scattering length $a_{\pi \mathrm{D}}$}

Evaluating the DGBT formula 10 , one obtains for the complex scattering length $a_{\pi \mathrm{D}}^{\mathrm{LO}}=[(-25.07 \pm 0.33)+i(6.23 \pm$ $0.26)] \cdot 10^{-3} m_{\pi}^{-1}$. Inserting these leading order values in eqs. 12 and 13 one obtains

$$
\begin{aligned}
& \operatorname{Re} a_{\pi \mathrm{D}}=0.010642 m_{\pi}^{-1} / \mathrm{eV} \cdot 0.9968 \cdot \epsilon_{1 s} \\
& \operatorname{Im} a_{\pi \mathrm{D}}=0.010642 m_{\pi}^{-1} / \mathrm{eV} \cdot 0.9989 \cdot\left(\Gamma_{1 s} / 2\right)
\end{aligned}
$$

with the factors 0.9968 and 0.9989 representing the "Trueman" correction. Consequently, our result for the $\pi \mathrm{D}$ scat- tering length reads

$a_{\pi \mathrm{D}}=\left[(-24.99 \pm 0.33)+i\left(6.22_{-0.26}^{+0.12}\right)\right] \times 10^{-3} m_{\pi}^{-1}$

Real and imaginary part of the $\pi \mathrm{D}$ scattering length $a_{\pi \mathrm{D}}$ as determined from the various measurements and including the Trueman correction are compared in table 8 .

The $\pi \mathrm{D}$ scattering length was studied recently within the $\chi \mathrm{PT}$ approach 60. In this work, almost prefect overlap is achieved of all constraints imposed on the $\pi N$ isoscalar and isovector scattering length $a^{+}$and $a^{-}$by pionic hydrogen and deuterium data. In addition, in a combined analysis using the preliminary results for shift and width in $\pi \mathrm{H}$ as given in 69 together with the new shift value in $\pi \mathrm{D}$ from this experiment, the uncertainty for $a^{+}$ could be reduced by a factor of two yielding a positive value by two standard deviations. By using the results for $a^{+}$and $a^{-}$from this combined analysis, $\operatorname{Re} a_{\pi \mathrm{D}}=$ $(-25.4 \pm 2.6) \times 10^{-3} m_{\pi}^{-1}$ is obtained.

The imaginary part was calculated in LO $\chi \mathrm{PT}$ to be $\operatorname{Im} a_{\pi \mathrm{D}}=(5.65 \pm 1.75) \cdot 10^{-3} m_{\pi}^{-1}[64$. One result of this work is that the real part induced by this absorptive contributions turns out to be $(7 \pm 2) \%$ of the total $\operatorname{Im} a_{\pi \mathrm{D}}$. It is much smaller than the usual naive estimate of $\operatorname{Re} a_{\pi \mathrm{A}}^{\text {induced }} \approx$ $\operatorname{Im} a_{\pi \mathrm{A}}$ [4, but can be traced back in the case of $\pi \mathrm{D}$ to cancellations of individually sizeable terms.

\subsection{Threshold parameter $\alpha$ in pion absorption}

The value derived from the $\pi \mathrm{D}$ hadronic width $\Gamma_{1 s}$ reads

$$
\alpha=\left(\begin{array}{r}
251 \\
+5 \\
-11
\end{array}\right) \mu \mathrm{b} .
$$

The central value differs by $1 \mu \mathrm{b}$ from the one given in ref. [70, because there the correction term $\delta_{\mathrm{D}}^{v a c}$ (see sect. 3.1] had not been considered. 
Table 8. Real and imaginary part $\operatorname{Re} a_{\pi \mathrm{D}}$ and $\operatorname{Im} a_{\pi \mathrm{D}}$ of the $\pi \mathrm{D}$ scattering length as extracted from atomic data including the Trueman correction $\sqrt{12}$ and $(13)$. Threshold parameter $\alpha$ for pion production and $\operatorname{Im} a_{\pi \mathrm{D}}$ are corrected for radiative capture and pair conversion $(20)$ but omitting any isospin breaking contributions. Production experiments reporting only the statistical uncertainty are marked $(*)$. Arrows $(\leftarrow / \rightarrow)$ indicate primary and derived quantities. The last line shows the result of a combined analysis of $\pi \mathrm{H}$ and $\pi \mathrm{D}$ data (see sect.6.5. Theoretical approaches are listed in chronological order.

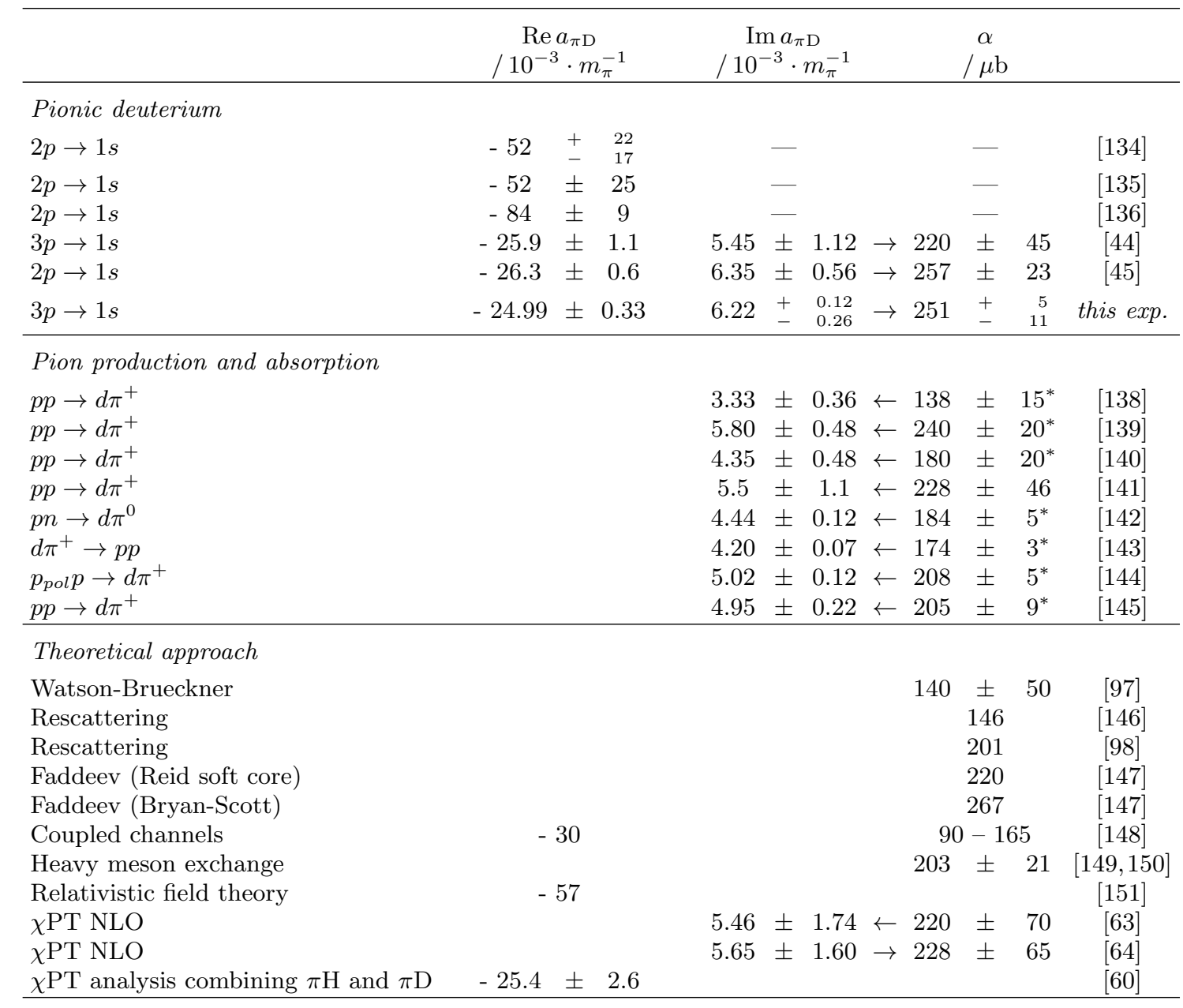

The theoretical understanding of $N N \leftrightarrow N N \pi$ reaction is continuously increasing. Within the approach of $\chi \mathrm{PT}$, a study of pion production including the terms in NLO yields $\alpha^{\mathrm{NLO}}=(220 \pm 70) \mu \mathrm{b} 63$ in good agreement with the pionic deuterium results (Table 8 and fig:14). The theoretical uncertainty is expected to decrease to below $10 \%$ within a few years from forthcoming NNLO calculations 137 .

The parameter $\alpha$ when determined from pion production experiments shows wide fluctuations even when comparing recent data. Often, only the statistical error is given for the cross section of the production experiments, but the fluctuations suggest significant systematic uncertainties, which may arise from uncertainties in the normalisation and/or Coulomb corrections. The expected order of magnitude of isospin breaking effects is about or less the precision of this experiment, which is far below the variation of the pion production data.

\subsection{Pion absorption on nucleon-nucleon pairs}

To describe the s-wave absorption strength on isoscalar $(\mathrm{I}=0)$ and isovector $(\mathrm{I}=1)$ nucleon-nucleon pairs $(N N)$, effective couplings $g_{0}$ and $g_{1}$ have been introduced representing the transitions ${ }^{3} S_{1}(I=0) \rightarrow{ }^{3} P_{1}(I=1)\left(g_{0}\right)$ and ${ }^{1} S_{0}(I=1) \rightarrow{ }^{3} P_{0}(I=1)\left(g_{1}\right)$ [152]. The transition strength $\left|g_{0}\right|^{2}$ is hence related to the imaginary part of the scattering length $a_{\pi \mathrm{D}}$ as determined from $\Gamma_{1 s}$ in $\pi \mathrm{D}$ by $\operatorname{Im} a_{\pi \mathrm{D}}=\frac{1}{3}\left(p_{n}^{*}\right)^{3}\left|g_{0}\right|^{2}\left(1+1 / S^{\prime}\right)[153]$ and, consequently, also to the threshold parameter $\alpha$ by

$$
\left|g_{0}\right|^{2}=\frac{1}{2 \pi m_{\pi}} \cdot \frac{p_{p}^{*}}{p_{n}^{*}} \cdot \frac{1}{p_{n}^{*}} \cdot \alpha
$$

(see sect.3.2. The CMS momentum of the neutrons acquired in the absorption reaction is $p_{n}^{*}=2.6076 m_{\pi}$.

The relative strength of the coupling for absorption on isoscalar to isovector $N N$ pairs was obtained to $\left|g_{0} / g_{1}\right|=$ 


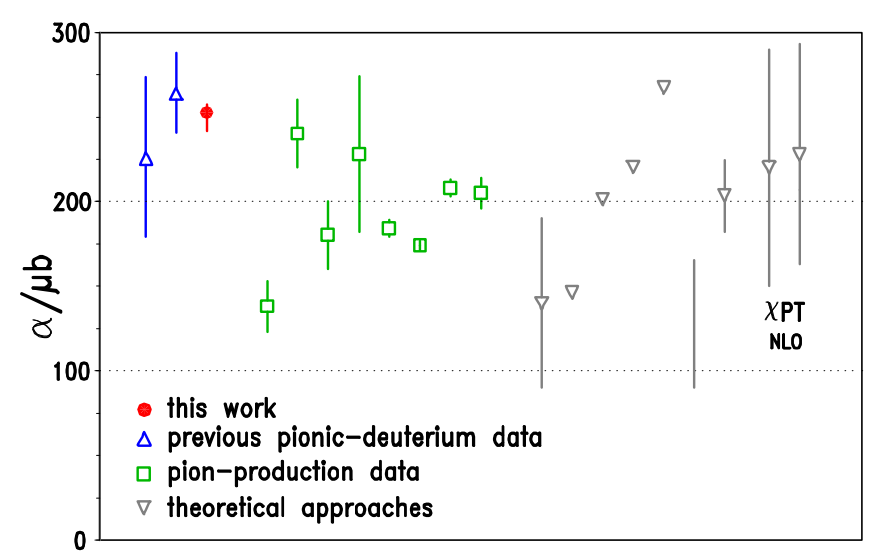

Fig. 14. Threshold parameter $\alpha$ for s-wave pion production compared to results from previous experiments and theoretical studies(from left). Previous X-ray measurements: 45, 44]; pion production and absorption: [138, 139, 140, 141, 142], [143, 144, 145]; Theoretical calculations: 97, 146, 98, 147, 147, 148, 149, 150, 63, 64. Points are given in the same order as listed in table 8

$2.48 \pm 0.24$ from the ratio of neutron-neutron to neutronprotons pairs emitted back-to-back after pion absorption in the helium isotopes [154,155]. From $\alpha$ as given by $\operatorname{Im} a_{\pi \mathrm{D}}$ and $\left|g_{0} / g_{1}\right|$ one obtains

$$
\begin{aligned}
& \left|g_{0}\right|=\left(2.780 \begin{array}{l}
+0.027 \\
-0.058
\end{array}\right) \cdot 10^{-2} m_{\pi}^{-2} \\
& \left|g_{1}\right|=(1.12 \pm 0.11) \cdot 10^{-2} m_{\pi}^{-2}
\end{aligned}
$$

where the effective coupling $g_{1}$ describes, e.g., the threshold transition strength of the reaction $p p \rightarrow p p \pi^{0}$ [156].

A phenomenological analysis for $N N \rightarrow N N \pi$ close to threshold using the ansatz $\sigma=B_{0} \eta^{2}$ [97] yields from fits to the cross section $B_{0}=27.6 \mu \mathrm{b}$. The uncertainty is estimated to be as large as $50 \%$ 157. However, the simple energy dependence is only valid in the limit of an infinitely large scattering length.

Scaling our result for $g_{1}$ one obtains a smaller value of $B_{0}=13.3 \pm 1.4 \mu \mathrm{b}$. This may be due to the fact that the extraction on both $\left|g_{0} / g_{1}\right|$ from $\pi^{-} H e$ absorption and $g_{0}$ from $\pi \mathrm{D}$ are affected differently by $N N$ final state interactions.

As discussed, e.g. by [158, 159], the phenomenological ansatz neglects the strong $N N$ final-state interaction leading to a completely different and complicated energy dependence close to threshold, in particular, when comparing $p n$ and $p p\left({ }^{1} S_{0}\right)$ final states 160,161 . This prevents a direct determination of the production amplitude from a simple parametrization of the cross section like $\propto \eta^{2}$.

Calculations including final-state and Coulomb interaction lead to values for $\sigma / \eta^{2}$ as small as $5 \mu \mathrm{b}$ when approaching threshold 158, 159]. This is in agreement with the long known suppression of the $p p \rightarrow p p \pi^{0}$ amplitude in LO [146] and a $\chi \mathrm{PT}$ calculation showing that loop contributions vanish up to NLO 162 .

\section{Summary}

The $\pi \mathrm{D}(3 p-1 s)$ X-ray transition in pionic deuterium has been studied using a high-resolution crystal spectrometer to determine the ground-state strong-interaction effects.

The accuracy of $1.3 \%$, obtained for the shift, supersedes the theoretical uncertainty of $9 \%$ achieved recently, and which can be traced back mainly to insufficient knowledge of low-energy constants. However, when used as a constraint for the pion-nucleon scattering lengths $a^{+}$and $a^{-}$as determined from pionic hydrogen, the new $\pi \mathrm{D}$ result reduces the uncertainty for the isoscalar scattering length $a^{+}$by a factor of about two yielding a non-zero and positive value 60 .

The accuracy achieved for the hadronic broadening of $4.2 \%$ reaches already the expected final uncertainty of (5$10) \%$ of forthcoming NNLO $\chi \mathrm{PT}$ calculations 137 . From its value, the transition strength $\alpha$ for s-wave pion production in nucleon-nucleon collisions is determined with unprecedented accuracy.

No line broadening by radiative de-excitation from non stabilised molecular states was identified which is corroborated from the study of the energy dependence of the $\pi \mathrm{D}(3 p-1 s)$ transition energy. However, weak evidence $(2 \sigma)$ was found for a Auger stabilised state.

Noteworthy, that at the $10 \%$ level no high-energy components could be identified stemming from low-lying Coulomb de-excitation transitions in contrast to pionic and muonic hydrogen.

\section{Acknowledgements}

We would like to thank N. Dolfus, B. Leoni, L. Stohwasser, and K.-P. Wieder for the technical assistance and V. Baru, C. Hanhart, A. Nogga, M. Hoferichter, and A. Rusetsky for continuous exchange of information on theoretical progress on the $\pi \mathrm{D}$ system. The Bragg crystal was manufactured by Carl Zeiss AG, Oberkochen, Germany. Partial funding and travel support was granted by FCT (Lisbon) and FEDER (Grant No. SFRH/BD/18979/2004 and project PTDC/FIS/102110/2008) and the Germaine de Staël exchange program. LKB is Unité Mixte de Recherche du CNRS, de l'École Normale Supérieure et de UPMC No. 8552. This work is part of the PhD thesis of one of us (Th. S., Univ. of Cologne, 2009).

\section{References}

1. S. Deser, M. L. Goldberger, K. Baumann, and W. Thirring, Phys. Rev. 96, 774 (1954).

2. D. Gotta, Prog. Part. Nucl. Phys. 52, 133 (2004).

3. T. E. O. Ericson and W. Weise, Pions and Nuclei, (Clarendon, Oxford, 1988) chapter 6.

4. K. A. Brückner, Phys. Rev. 98, 769 (1955).

5. J. Gasser, V. E. Lyubovitskij, and A. Rusetsky, Phys. Rep. 456, 167 (2008).

6. M. Lüscher, Commun. Math. Phys 105, 153 (1986); Nucl. Phys. B 354, 531 (1991). 
7. A. Torok et al., Phys. Rev. D 81, 074506 (2010).

8. S. R. Beane, W. Detmold, K. Originos, and M. J. Savage, Prog. Part. Nucl. Phys. 66, 1 (2011).

9. S. Weinberg, Phys. Rev. Lett. 17, 616 (1966).

10. Y. Tomozawa, Nuovo Cim. A 46, 707 (1966).

11. J. Gasser and H. Leutwyler, Phys. Rep. 87, 77 (1982).

12. A. W. Thomas and W. Weise, The Structure of the Nucleon, (WILEY-VCH, Berlin, 2001) chapter 7.

13. S. Weinberg, Physica 96A, 327 (1979).

14. J. Gasser and H. Leutwyler, Ann. Phys. 158, 142 (1984).

15. J. Gasser and H. Leutwyler, Nucl. Phys. B 250, 465 (1985).

16. G. Ecker, Prog. Part. Nucl. Phys. 35, 1 (1995).

17. V. Bernard, N. Kaiser, and Ulf-G. Meißner, Int. J. Mod. Phys. E 4, 193 (1995).

18. S. Scherer, Introduction to Chiral Perturbation Theory, Adv. Nucl. Phys. 27, 277 (2003) (arXiv:hep$\mathrm{ph} / 0210398 \mathrm{v} 1$ ).

19. V. Bernard, Prog. Part. Nucl. Phys. 60, 82 (2008).

20. T. Becher and H. Leutwyler, J. High En. Phys. 06, 017 (2001).

21. J. Gasser, H. Leutwyler, and M. E. Sainio, Phys. Lett. B 253, 252 (1991).

22. M. E. Sainio, Pion-nucleon $\sigma$-term - a review, in Proc. of the $9^{\text {th }}$ Symp. on Meson-Nucleon Physics and the Structure of the Nucleon (MENU'01), Washington D. C., 2001, $\pi N$ newsletter 16, 138 (2002), ISSN 0942-4148, and references therein.

23. M. L. Goldberger, H. Miyazawa and R. Oehme, Phys. Rev. 99, 986 (1955).

24. T.E. O.Ericson, B. Loiseau and A.W. Thomas, Phys. Rev. C 66, 014005 (2002).

25. V. V. Abaev, P. Metsä, and M. E. Sainio, Eur. Phys. J. A 32, 321 (2007).

26. V. Bernard, N. Kaiser, and Ulf-G. Meißner, Phys. Lett. B 383, 116 (1996).

27. M. A. Kovash et al., $\pi N$ newsletter 12, 51 (1997).

28. O. Hanstein, D. Drechsel, and L. Tiator, $\pi N$ newsletter 12, 56 (1997).

29. D. F. Measday, Phys. Rep. 354, 243 (2001) .

30. T. Gorringe and H. W. Fearing, Rev. Mod. Phys. 76, 31 (2004).

31. V. A. Andreev et al., Phys. Rev. Lett. 99, 032002 (2007).

32. D. Sigg et al., Nucl. Phys. A 609, 310 (1996).

33. V. E. Lyubovitskij and A. Rusetsky, Phys. Lett. B 494, 9 (2000).

34. J. Gasser et al., Eur. Phys. J. C 26, 13 (2002).

35. P.Zemp, Proc. of Chiral Dynamics 2003, p.128, Bonn, Germany, September 8-13, 2003, arXiv:hepph/0311212v1; Pionic Hydrogen in QCD+QED: Decay width at NNLO, Ph. D. Thesis, University of Bern (2004).

36. G. Rasche and W. S. Woolcock, Nucl. Phys., A 381, 405 (1982).

37. J. Spuller et al., Phys. Lett. 67 B, 479 (1977).

38. G. C. Oades, G. Rasche, W.S. Woolcock, E. Matsinos, A. Gashi, Nucl. Phys. A 794, 73 (2007).

39. T. E. O. Ericson and W. Weise, Pions and Nuclei, (Clarendon, Oxford, 1988) chapter 9.

40. ibidem, chapter 4.

41. A. W. Thomas and R.H. Landau, Phys. Rep. B 58, 121 (1980).

42. A.Deloff, Fundamentals in Hadronic Atom Theory, (World Scientific, London, 2003) chapter 15.
43. D. Sigg et al., Nucl. Phys. A 609, 269 (1996).

44. D. Chatellard et al., Phys. Rev. Lett. 74, 4157 (1995); Nucl. Phys. A 625, 855 (1997).

45. P. Hauser et al., Phys. Rev. C 58, R1869 (1998).

46. H.-Ch. Schröder et al., Eur. Phys. J C 21, 473 (2001).

47. S. Weinberg, Phys. Lett. B 295, 114 (1992).

48. V. Baru and A.E. Kudryavtsev, Phys. of At. Nucl. 60, 1475 (1997).

49. S. R. Beane, V. Bernard, T.-S. Lee, and U.-G. Meißner, Phys. Rev. C 57, 424 (1998).

50. V.E. Tarasov, V. V. Baru, and A. E. Kudryavtsev, Phys. At. Nucl. 63, 801 (2000).

51. A. Deloff, Phys. Rev. C 64 (2001) 065205.

52. N. Kaiser, Phys. Rev. C 65, 057001 (2002).

53. B. Burasoy and H. W. Grieshammer, Int. J. Mod. Phys. E 12, 65 (2003).

54. S. R. Beane, V. Bernard, E. Epelbaum, U.-G. Meißner, and D. R. Phillips, Nucl. Phys. A 720, 399 (2003).

55. M. Döring, E. Oset, and M. J. Vicente Vacas, Phys. Rev. C 70, 045203 (2004).

56. B. F. Irgaziev and B. A. Fayzullaev, arXiv:hepph/0404203v1 (2004).

57. U.- G. Meißner, U. Raha, and, A. Rusetsky, Eur. Phys. J. C 41, 213 (2005); Eur. Phys. J. C 45, 545 (2006).

58. U.- G. Meißner, U. Raha, and, A. Rusetsky, Phys. Lett. B 639, 478 (2006).

59. M. Hoferichter, B. Kubis and U.-G. Meißner, Phys. Lett. B 678, 65 (2009); Nucl. Phys. A 833,18 (2010).

60. V. Baru et al., Phys. Lett. B 694, 473 (2011) (arXiv:nuclth/1003.4444v2).

61. M. Hoferichter, B. Kubis and U.-G. Meißner, PoS CD09, 014 (2009) (arXiv:hep-ph/0910.0736).

62. J. Hüfner, Phys. Rep. 21, 1 (1975).

63. V. Lensky et al., Eur. Phys. J. A 27, 37 (2006).

64. V. Lensky et al., Phys. Lett. B 648, 46 (2007).

65. V. L. Highland et al., Nucl. Phys. A 365, 333 (1981).

66. D. W. Joseph, Phys. Rev. 119, 805 (1960).

67. R. MacDonald et al., Phys. Rev. Lett. 38, 746 (1977).

68. PSI proposal R-98-01, www2.fz-juelich.de/ikp/exoticatoms.

69. D. Gotta et al., Lect. Notes Phys. 745, 165 (2008).

70. Th. Strauch et al., Phys. Rev. Lett. 104, 142503 (2010).

71. F.J.Hartmann, Proceedings of Physics of Exotic Atoms on Electromagnetic Cascade and Chemistry, Erice, Italy, 1989 (Plenum Press, New York, 1990) p. 23 and p. 127, and references therein.

72. J.S. Cohen, Rep. Prog. Phys. 67, 1769 (2004).

73. E. Borie and M. Leon, Phys. Rev. A 21, 1460 (1980).

74. M. Leon and H. A. Bethe, Phys. Rev. 127, 636 (1962).

75. R. al Hassani et al., Z. Phys. A 351, 113 (1995).

76. T. S. Jensen and V. E. Markushin, Eur. Phys. J. D 19, 165 (2002).

77. T. S. Jensen and V. E. Markushin, Eur. Phys. J. D 21, 261 (2002).

78. T. S. Jensen and V. E. Markushin, Eur. Phys. J. D 21, 271 (2002).

79. L. Bracci and G. Fiorentini, Nuovo Cim. A 43, 9 (1978).

80. R. Pohl et al., Phys. Rev. Lett. 97, 193402 (2006).

81. J. B. Czirr et al., Phys. Rev. 130, 341 (1963).

82. A. Badertscher et al., Europhys. Lett. 54, 313 (2001), and references therein.

83. D. S. Covita et al., Phys. Rev. Lett. 102, 023401 (2009). 
84. M. Hennebach, Precision measurement of ground state transitions in pionic hydrogen, $\mathrm{Ph}$. D. thesis, Universität zu Köln (2003), http://kups.ub.uni-koeln.de/744/

85. V. N. Pomerantsev and V.P. Popov, Phys. Rev. A 73, 040501(R) (2006).

86. T.S. Jensen, V. N. Pomerantsev, and V.P. Popov, arXiv:nucl-th/0712.3010v1 (2007).

87. V.P.Popov and V.N.Pomerantsev, arXiv:nuclth/0712.3111v1 (2007).

88. D. Taqqu, AIP Conf. Proc. 181, 217 (1989).

89. S. Jonsell, J. Wallenius, P. Froelich, Phys. Rev. A 59, 3440 (1999).

90. R. Pohl, Hyperfine Int. 193, 115 (2009).

91. E. Lindroth, J. Wallenius, S. Jonsell, Phys. Rev. A 68, 032502 (2003); Phys. Rev. A 69, 059903(E) (2004).

92. S. Kilic, J.-P. Karr, L. Hilico, Phys. Rev. A 70, 042506 (2004).

93. T. L. Trueman, Nucl. Phys. 26, 57 (1961).

94. E. Lambert, Helv. Phys. Acta 42 (1969) 667; ibidem 43, 713 (1970).

95. J. Mitroy and I. A. Ivallov, J. Phys. G 27, 1421 (2001).

96. D. Eiras and J. Soto, Phys. Lett. B 491, 101 (2000).

97. A. H. Rosenfeld, Phys. Rev. 96, 139 (1954).

98. A. Reitan, Nucl. Phys. B 11, 170 (1969).

99. H. Machner and J.Niskanen, Nucl.Phys. A 776, 172 (2006).

100. K. Brückner, R. Serber, and K. Watson, Phys. Rev. 81, 575 (1951).

101. V. Baru, C. Hanhart, and A. Rusetski, priv. comm.

102. A. Filin et al., Phys. Lett. B 681, 423 (2009).

103. E. Byckling and K. Kajantie, Particle Kinematics, John Wiley and Sons, London (1973) chapter II.6.

104. L. M. Simons, Physica Scripta T22, 90 [1988).

105. L. M. Simons, Hyperfine Int. 81, 253 (1993).

106. D. S. Covita et al., Rev. Scient. Instr. 79, 033102 (2008).

107. J. Eggs and K. Ulmer, Z. angew. Phys., 20(2), 118 (1965).

108. G. Zschornack, Nucl. Instr. Meth. 200, 481 (1982).

109. N. Nelms et al., Nucl. Instr. Meth. A 484, 419 (2002).

110. G. Basile et al., Phys. Rev. Lett. 72, 3133 (1994).

111. R. Deslattes et al., Rev. Mod. Phys., vol. 75, no. 1, 35 (2003).

112. P. Indelicato et al., Rev. Sci. Instrum. 77, 043107 (2006).

113. D. F. Anagnostopulos et al., Nucl. Instr. Meth. A 545, 217 (2005).

114. M. Trassinelli et al., J. Phys., Conf. Ser. 58, 129 (2007).

115. D. S. Covita, High-precision spectroscopy of the $3 p-1 s$ transition in muonic hydrogen, $\mathrm{Ph}$. D. thesis, University of Coimbra (2008), http://hdl.handle.net/10316/7521.

116. M. Sanchez del Rio and R. J. Dejus, Proc. SPIE Int. Soc. Opt. Eng. 3448, 246 (1998); ibidem 5536, 171 (2004); http://www.esrf.eu/computing/scientific/xop2.1

117. Th.Strauch, High-precision measurement of stronginteraction effects in pionic deuterium, Ph. D. thesis, Universität zu Köln (2009), http://kups.ub.unikoeln.de/2813/

118. WM. J. Veigele, At. Data Tables 5, 51 (1973).

119. M. O. Krause and J. H. Oliver, J. Phys. Chem. Ref. Data 8, 329 (1979)

120. T. Mooney, Argonne National Laboratory, priv. comm. (2007).

121. B. L. Henke, E. M. Gullikson, and J. C. Davies, At. Data Nucl. Data Tables 54, 181 (1993).

122. C. T. Chantler, J. Phys. Chem. Ref. Data 24, 71 (1995).
123. F. N. Chukhovskii, G. Hölzer, O. Wehrhan, and E. Förster, J. Appl. Cryst. 29, 438 (1998).

124. F. Cembali et al., J. Appl. Cryst. 24, 424 (1992).

125. F. James and M. Roos, Comput. Phys. Commun. 10, 343 (1975).

126. U. C. Bergmann and K. Riisager, Nucl. Instrum. Meth. A 489, 444 (2002).

127. C. Amsler et al. (PDG 2008), Phys. Lett. B 667, 1 (2008).

128. R. Pohl et al., Nature 466, 213 (2010).

129. C. G. Parthey et al., Phys. Rev. Lett. 104, 233001 (2010).

130. Y. Lu and R. Rosenfelder, Phys. Lett. B 319, 7 (1993); Phys. Lett. B 333, 564(E) (1994).

131. W. Leidemann and R. Rosenfelder, Phys. Rev. B 51, 427 (1995).

132. J. Ahrens et al., Eur. Phys. J. A 23, 113 (2005).

133. R. Koch, Nucl. Phys. A 448, 707 (1986).

134. J. Bailey et al., Phys. Lett. 50 B, 403 (1974).

135. E. Bovet et al., Nucl. Instr. Meth. 190, 613 (1981).

136. E. Bovet et al., Phys. Lett. 153 B, 231 (1985).

137. C. Hanhart, priv. comm. (2010).

138. F. S. Crawford and M. L. Stevenson, Phys. Rev. 97, 1305 (1955).

139. C. M. Rose, Phys. Rev. 154, 1305 (1967).

140. C. Richard-Serre et al., Nucl. Phys. B 20, 413 (1970).

141. D. Aebischer et al., Nucl. Phys. B 108, 214 (1976).

142. D. A. Hutcheon et al., Phys. Rev. Lett. 64, 176 (1990); Nucl. Rev. A 535, 618 (1991).

143. B. G. Ritchie et al., Phys. Rev. Lett. 66, 568 (1991).

144. P. Heimberg et al., Phys. Rev. Lett. 77, 1012 (1996).

145. M. Drochner et al., Nucl. Phys. A 643, 55 (1998).

146. D. S. Koltun and A. Reitan, Phys. Rev. 141, 1413 (1966).

147. I. R. Afnan and A.W. Thomas, Phys. Rev. C 10, 109 (1974).

148. C. Fayard, G. H. Lamot, and T. Mizutani, Phys. Rev. Lett. 45, 524 (1980).

149. C. J. Horowitz, Phys. Rev. C 48, 2920 (1993).

150. J. A. Niskanen, Phys. Rev. C 53, 526 (1996).

151. A. N. Ivanov et al., Z. Phys. A 358, 81 (1997).

152. S. G. Eckstein, Phys. Rev. 129, 413 (1963).

153. J.-F. Germond and C. Wilkin, J. Phys. G 14, 181 (1988).

154. D. Gotta et al., Phys. Rev. C 51, 469 (1995).

155. E. Daum et al., Nucl. Phys. A 589, 553 (1995).

156. J. Roginsky and C. Werntz, Phys. Rev. C 40, 2732 (1989).

157. D. G. Long, M. Sternheim, and R. R. Silbar, Phys. Rev C 26, 586 (1982).

158. G. A. Miller and P. Sauer, Phys. Rev. C 44, R1725 (1991).

159. H. O. Meyer et al., Nucl. Phys. A 539, 633 (1992).

160. C. Hanhart, Phys. Rep. 397, 155 (2004).

161. V. Baru et al., Phys. Rev. C 80, 044003 (2009).

162. C. Hanhart and N. Kaiser, Phys. Rev. C 66, 054005 (2002). 